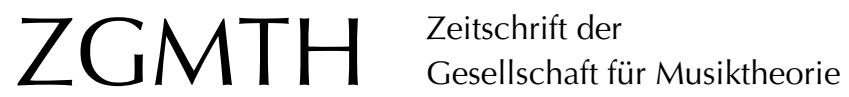

Jeßulat, Ariane (2015): Synchron und diachron. Zum Zusammenhang zwischen Kontrapunkt und Prozessualität in romantischer Formensprache. ZGMTH 12/1, 99-128. https://doi.org/10.31751/803

(C) 2015 Ariane Jeßulat

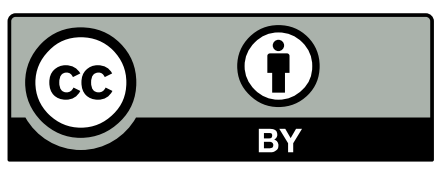

Dieser Text erscheint im Open Access und ist lizenziert unter einer Creative Commons Namensnennung 4.0 International Lizenz.

This is an open access article licensed under a Creative Commons Attribution 4.0 International License.

veröffentlicht / first published: 23/07/2016

zuletzt geändert / last updated: 26/09/2017 


\title{
Synchron und diachron Zum Zusammenhang zwischen Kontrapunkt und Prozessualität
in romantischer Formensprache
}

Ariane Jeßulat

\begin{abstract}
Der Beitrag untersucht die für romantische Formensprache etablierte Theorie der `organischen Form` (১organic metaphor`). `Organische Form` wird implizit wie explizit mit metaphorischen Konnotationen verwendet, die in der Anwendung auf die Werkanalyse Voreinstellungen mit sich bringen. Damit einher geht eine unscharfe Überblendung von Produktions- und Rezeptionsästhetik sowie eine seit den Anfängen klassisch-romantischer Formenlehre fast selbstverständliche Abkehr des Interesses von kontrapunktischen Techniken, die der Illusion spontaner Einfälle und natürlicher Prozesse zuwiderlaufen können. An Hand dreier Einzelanalysen aus Werken von Schumann, Brahms und Mendelssohn unter der begleitenden kritischen Lektüre von Johann Christian Lobes Lehrbuch der musikalischen Komposition werden Fälle von Prozessualität herausgearbeitet, deren Verlauf und Dramaturgie sich der herkömmlichen Metaphorik von sorganischer Form kommen intakt sind.
\end{abstract}

This article discusses 'organic metaphor', a well-established theory of form with regard to the Romantic idiom. 'Organic form' is implicitly and explicitly used with metaphorical connotations that imply a preselection when applied to musical analysis. This goes hand in hand with a diffuse blending of the aesthetics of production and reception, as well as with an almost matter of course renunciation of contrapuntal techniques, typical of the Classic-Romantic Formenlehre, which could conflict with the illusion of spontaneous inspiration and natural processes. Analyses of three works by Schumann, Brahms and Mendelssohn together with a critical reading of Johann Christian Lobe's Lehrbuch der musikalischen Komposition are used to demonstrate cases of processuality, whose development and dramaturgy defy the conventional imagery of 'organic form' despite remaining completely intact as musically functional contexts.

Besonders für die Analyse des klassisch-romantischen Repertoires ist kaum eine Begrifflichkeit verbreiteter als die, welche auf der Vorstellung von einer >organischen Form fußt. Dabei knüpft die in den dokumentierten Quellen nie ganz homogene Ästhetik der >organischen Form schichte allein von der Goethe-Zeit bis zur kritischen Aufbereitung durch die DahlhausSchule mehrere Paradigmenwechsel, tendenziöse Verschärfungen, aber auch wieder wissenschaftliche Redaktionen erfahren hat. Dementsprechend unterschiedliche Bedeutungen werden in der musikalischen Analyse oft anachronistisch nebeneinander verwendet. Dieser Beitrag versucht an Hand einer nur kleinen Auswahl von Quellen, nämlich 
der Kompositionslehre von Johann Christian Lobe aus der Mitte des 19. Jahrhunderts und einiger weniger Texte von Carl Dahlhaus, auf Probleme der musikalischen Analyse aufmerksam zu machen, die dann entstehen, wenn die zu analysierende Musik zwar die Prämisse des ıorganischen Zusammenhangs des Ganzen zu den Teilen Funktionszusammenhangs klar erfüllt, dabei sich aber einer Einordnung in den metaphorischen Überschuss der Vorstellungen von >organischer Form ‘ ebenso klar entzieht, also weder offensichtlich wachsende, drängende oder anthropomorph-psychologische Analoga findet noch diese dialektisch in komplexeren Formprozessen anstrengt. Dabei geht es kaum um eine Korrektur oder gar ein Widerlegen etablierter und äußerst tragfähiger formanalytischer Ansätze aus dem Vorstellungsfeld prozessualer Logik wie der sentwickelnden Variation oder der skontrastierenden Ableitung`, deren Rezeption nicht nur die Geschichte der Musiktheorie, sondern immer wieder auch die Geschichte der kompositorischen Praxis selbst durchsetzt, sondern um das Aufdecken unbemerkt und unbewusst waltender metaphorisch gebundener Vor-Urteile, deren Wirkung sowohl potentielle Analyseergebnisse beeinflusst als auch die Auswahl dessen, was überhaupt unter der Prämisse der >organischen Form` analysiert wird.

\section{Organische Form, entwickelnde Variation ${ }^{1}$}

In der romantischen Formästhetik sind Denkmodelle nach dem Vorbild organischer Prozesse zentral. Zum weiten Assoziationsfeld der natura naturans gehören Termini wie 'Keimzelle`, 'Erfindungskern`, sStau oder in anderem Zusammenhang aus der romantischen Naturwissenschaft und Anthropologie entlehnte Wachstumsmetaphern mit großer Selbstverständlichkeit. ${ }^{2}$ Neben dem direkten wissenschaftlichen Interesse, Naturwissenschaft und philosophische Ästhetik unter einer gemeinsamen metaphysischen Leitidee zusammenzufassen, ist es bis heute ${ }^{3}$ der evidente Erfolg derartiger analytischer Methoden, der auch die begleitende Metaphorik wirksam erhält. In der Mehrzahl der Fälle ist es ausgesprochen sinnvoll, so etwas wie Organik im Sinne von Werkästhetik durch das stimmige Verhältnis des Ganzen zu seinen Teilen an Hand motivischer Entwicklungspro-

1 Vgl. die Zusammenfassung bei Clemens Kühn (1993, 224): „Der Zusammenhang von Musik ist gewährleistet durch Logik in der Gestaltung, Aufeinanderfolge und Verbindung ihrer Gedanken. Ausgangspunkt ist ein individueller musikalischer Gedanke. Er wirkt sich aus, und sein Einfluß reicht bis weit in ein Werk hinein. Diese Folgerungen tragen die Musik.»

2 Zum unscharfen Übergang zwischen psychologischen und biologisch-naturwissenschaftlichen Metaphern unter dem Dach einer Analogie zwischen Kunstwerk und Leben vgl. Danuser 2014, 309 : » [...] the notion of stransformation ‘ [...] involves more basic principles than just those relevant to twentieth-century music. As a feature of variation, it may be used in the sense of altering a given musical structure of whatever kind or size into a different one - be it in reference to thematic processes in variations [...] as well as in structures following the idea of sdeveloping variation<, or be it understood as a general aesthetic principle derived from analogy between life and art, as stipulated around 1900 by Gustav Mahler who would not allow unaltered repetition of any kind in music."

3 Innerhalb der Metapher >organische Form ‘ haben seit ihrem Aufkommen allerdings diverse differenzierende Paradigmenwechsel stattgefunden. Die entschiedenste Hinwendung zu einem Verständnis von Form als energetisch dynamischem Prozess fand nicht im 19. Jahrhundert selbst, sondern zu Beginn des 20. Jahrhunderts statt, fließt aber aus heutiger Sicht gelegentlich anachronistisch in die interpretierende Lektüre älterer Quellen mit ein (vgl. Borio 2011, 149). 
zesse zu belegen, zu identifizieren und so im analytischen Nachvollzug mit dem Werk selbst in Kontakt zu treten. Schlüsseltexte der Philosophie, Literatur- und Naturwissenschaft gehen dabei in die Musikästhetik des 19. Jahrhunderts ein, sei es, dass Goethes Metamorphose der Pflanzen ${ }^{4}$ zeitgenössische wie jüngere Formenlehren beeinflusst, sei es, dass die Einleitung zu Hegels Phänomenologie des Geistes sich einer entsprechenden Knospen-Metapher bedient ${ }^{5}$ oder dass in der Analyse sinfonischer Formen Begriffe wie sentwickelnde Variation und `Trieb، auf derselben kategorialen Ebene eingesetzt werden.

Durch die Problematik einer vornehmlich an Beethoven geschulten Formenlehre, deren Exempla aus Beethovens mittlerer Periode das ästhetische Postulat organischen Wachstums tatsächlich bis zu einem gewissen Grade erfüllen, ist die aktuelle musikwissenschaftliche Forschung schon seit längerem für die Einseitigkeit und die Ideologisierung solcher >organischer, Formtheorien sensibilisiert.

Dennoch ist nach wie vor Wachsamkeit vor stillschweigenden, möglicherweise nicht intendierten metaphorischen Implikationen geboten ${ }^{6}$, wenn es um den detaillierten musikalischen Nachvollzug solcher eventuell nicht ganz erloschener Katachresen ${ }^{7}$ im Dienste einer zunächst nicht wertend gemeinten Formanalyse geht.

So ist es im Sinne einer musikgeschichtlichen Würdigung in vieler Hinsicht zu rechtfertigen, Johann Christian Lobes Kompositionslehre ${ }^{8}$ als Meilenstein romantischer Formenlehre in ein kanonisierendes Lehrwerk aufzunehmen.

Lobe's emphasis on creating musical cells that can be endlessly elaborated also reveals another agenda increasingly featured in theories of form: the urge to make the organic metaphor more and more palpable in analysis. Apart from the idea of motives as seeds, one of the principal methodological manifestations of the organicist perspective is the analysis of formal functions. Musical form is here figured as an organism in which every part has a specific function. ${ }^{9}$

Bei aller Relevanz des analytischen Paradigmenwechsels im Vergleich zu Kochs sAnlage`, der aus Lobes Texten spricht, wäre es allerdings zum Erfassen der konkreten Pro-

4 Hier vor allem mit einer impliziten Nähe zum Prometheus-Mythologem (vgl. Goethe 1977, 70-119).

5 Die Passage aus der Vorrede lautet (Hegel 1987, 10): „Die Knospe verschwindet in dem Hervorbrechen der Blüte, und man könnte sagen, daß jene von dieser widerlegt wird, ebenso wird durch die Frucht die Blüte für ein falsches Dasein der Pflanze erklärt, und als ihre Wahrheit tritt jene an die Stelle von dieser. Diese Formen unterscheiden sich nicht nur, sondern verdrängen sich auch als unverträglich miteinander. Aber ihre flüssige Natur macht sie zugleich zu Momenten der organischen Einheit, worin sie sich nicht nur nicht widerstreiten, sondern eins so notwendig als das andere ist, und diese gleiche Notwendigkeit macht erst das Leben als Ganzes aus."Vgl. dazu den Kommentar in Ludwig 1997, 39.

6 Zur impliziten Metaphorizität musikalischer Analyse vgl. Thorau 2012. Thorau spricht von einem aktuellen Paradigmenwechsel in der Forschung »in den vergangenen zwei Jahrzehnten« (ebd., 26-27).

7 Vgl. Rohringer 2014, 164: „Sie [einige musiktheoretische Termini] sind zu Katachresen versunkene Metaphern, die sich von einer buchstäblichen Redeweise nicht mehr abstoßen. Gleichwohl ist es möglich, sie zurück an die Oberfläche zu holen, werden entsprechende Kontexte geschaffen. "Siehe auch die kommentierte Auswahlbibliographie zu diesem Thema in Rohringer 2014, 182-184.

8 Lobe 1858.

9 Burnham 2002, 892. 
bleme von Organismusmetaphern im Hinblick auf Musik - und zwar nicht nur technischer, sondern ebenso ästhetischer Natur - wesentlich interessanter zu untersuchen, welche Bereiche von Lobes Ansatz gerade nicht funktionieren, und wo er den eigenen Anspruch, das kreative Potential organischer Form didaktisch zu reduzieren, nicht ansatzweise erfüllen kann. ${ }^{10}$

Zunächst sollte zu Burnhams Ausführungen ergänzt werden, dass Lobes Intention nicht primär die Analyse ist (»to make organic metaphor more and more palpable for analysis«), sondern das eigene Schaffen: Lobes Kompositionslehre ist ein um 1850 hochmoderner Lehrgang in musikalischer sinventios, und zwar am Leitfaden der Struktur seiner Produkte, d. h. am Leitfaden der organischen Form selbst. Der Schüler soll das, was er komponiert, analog im Schaffensprozess erleben: ${ }^{11}$

Wenn man daher den Studiengang so einrichten kann, dass die Übungen alle, gleich von Anfang herein, wirkliche kleine Tonbilder werden, die sich mit den stufenweise weiter erschlossenen Regeln auch weiter ausspinnen, aneinanderreihen, und zuletzt zu ganzen Tonstücken zusammenfügen und ausbilden, so wird des Jüngers Lernlust nicht allein nicht ermatten, sondern im Gegenteil sich immer beleben und steigern. ${ }^{12}$

\section{Erlebnisqualität und literarisches Ethos}

Lobes pädagogische Entscheidung, die musikalische Ausbildung von Anfang an nach dem Ideal von Meisterschaft zu gestalten, entspricht den pädagogischen Ansätzen seiner Zeit. So steht Goethes Idee eines ganzheitlichen und stufenweisen Lernens am Vorbild der Natur ${ }^{13}$ hier ebenso Pate wie das in Musikvereinen und in einer blühenden Musikkritik gewachsene neue Selbstbewusstsein musikalischer Amateure, aus dem die Forderung spricht, zwischen das eigene Verstehen und Empfinden und die Meisterwerke keinerlei tradierte und überkommene Handwerkslehre treten zu lassen. ${ }^{14}$

10 Vgl. als kritisches Gegenstück zeitgenössische spöttische Kritiken auf Werke, deren entwickelndes Festhalten an einer Keimzelle zwar deutlich und intendiert, aber nicht überzeugend ist: z. B. von Robert Schumann (1982, 25-28) oder Adolf Bernhard Marx (1925, 399), der gehässig-lustvoll Ludwig Bergers zweite Klaviersonate verreißt, die bewusst nur über eine einzige musikalische Arabeske komponiert ist: „Man erzählt: ein Kandidat des Predigtamtes habe sich anheischig gemacht, über jedes gegebene Thema aus dem Stegreife zu reden: der Kirchenpatron habe ihm statt des erwarteten Textes ein leeres Blatt auf die Kanzel geschickt und der Redner, es von allen Seiten wendend, sich sogleich den Text: Hier ist nichts und da ist nichts; aus Nichts hat Gott die Welt erschaffen - daraus aber eine gute Predigt entsponnen."

11 Vgl. Stefan Rohringers Beobachtung zu einer metaphorisch gelenkten Analyse, deren standardisierter Verlauf auch literarisch die Form des Stückes nachzufahren scheint $(2014,174)$.

12 Lobe 1858, Vf.

13 Im Sinne des ganzheitlichen Stufenlehrgangs der fiktiven »pädagogischen Provinz« im zweiten Buch des Romans Wilhelm Meisters Wanderjahre (Goethe 1982, 165-184).

14 Vgl. Gottfried Webers genialistische Bemerkung über »leidige Generalbassschulen« (Weber 1817, Vorrede [2]) und Lobe (1858, VII): „Dasselbe würde es sein, wenn man des jetzigen Schülers Einbildungskraft, anstatt mit den blühenden Bildern unserer großen modernen Meister zuerst mit den Gedankenformen der Fuge und des gebundenen Stils besäete.« 
So ist ein Zusammengehen von Formästhetik und Kontrapunkt, wie es in diesem Beitrag untersucht werden soll, im Hinblick auf das Empfinden der romantischen Zeitgenossen wie auf das damals geläufige literarische Idiom des Musikschrifttums absurd, da 'Kontrapunkt in der einschlägigen Literatur zum Stigma der Unmusikalischen, der Nicht-Eingeweihten, der Nicht-Enthusiasten gehört. So lässt sich E. T. A. Hoffmanns Alter Ego Ritter Gluck auf das Gespräch mit dem Erzähler erst dann ein, als dieser den falschen Eindruck er sei ein »Oktavenjäger", der seine professionelle Kennerschaft nach außen trägt, durch das Bekenntnis, er sei ein begeisterungsfähiger Laie, tilgt. ${ }^{15}$ Derartige Figuren, aus denen Hoffmann selbst spricht, geben ebenso den Überdruss am Handwerk und am Metier wieder wie einige literarische Tropen, die Hoffmann vor allem geprägt hat und die zum stilistischen Rüstzeug der musikalischen Kritik im 19. Jahrhundert gehören. Im Falle der Ästhetik der >organischen Form، könnte man sogar behaupten, dass diese in letzter Konsequenz ohne die Integration in hochmetaphorische Literatur älteren und jüngeren Datums eine deutlich andere, möglicherweise kritischere und adäquatere Rezeption erfahren hätte.

Die Prosa der paraphrasierenden ${ }^{16}$ Texte der romantischen Musikzeitschriften und ihre zum Teil unscharfe Trennung zwischen wissenschaftlicher Erörterung und literarischem Essay bis weit in den Bereich der Fiktion hinein unterliegen schon lange einer historisch informierten und sinnvoll distanzierenden Aufarbeitung. Es steht außer Frage, dass Begriffe wie `Naturı, 'Schönheit‘, ‘Werkı, ’Gänze` oder `Trieb` nicht nur Metaphern, sondern zudem historisch kontingent sind. Allerdings scheinen auch aktuell gebräuchliche, mit prozesshaft-anthropomorphen Vorgängen verbundene Metaphernsysteme, in denen vor allem die Personifizierung der zu analysierenden Musik eine wesentliche Rolle spielt, eine ähnliche Vorrangstellung des Erlebnisses herauszuarbeiten, auch wenn dieser jüngere, sogenannte kognitivistische Ansatz durch psychologische und empirische Studien wissenschaftlich relativiert wird. ${ }^{17}$

Edward T. Cones persona-Konzept ${ }^{18}$ ist ein Beispiel dafür, wie das romantische Ideal der natura naturans als Schöpferkraft in ein idealisiertes Subjekt durch das Hören transformiert wird, eine Art rezipierendes >lyrisches Ichı, das das Werk durch sich hindurchgehen lässt und dessen Eindrücke nach einer wissenschaftlich-terminologischen Klärung die Basis für die analytische Arbeit bilden. Die Gemeinsamkeit mit Marx, Brendel, Lobe und der sneudeutschen Schule insgesamt liegt in der Forderung nach expressiven Inhalten und Wirkungen, die ein nacherlebend analysierender Hörer - nach Cone - nur durch Identifikation mit einer persona empfinden kann, so dass die persona zum heuristischen Werkzeug der Analyse wird. charakteristisch war, gleicht einer [um 1970] gerade vergangenen Mode, die zum Spott herausfordert, aber noch nicht lange genug veraltet ist, um zum Objekt historischer Gerechtigkeit zu werden.« Dahlhaus 1970, $24 \mathrm{ff}$.

17 Ein Beispiel für einen kritischen und reflektierten, dabei aber vor allem werknahen Umgang mit dem kognitivistischen Ansatz ist Bolay 2013.

18 Cone 1974. 
Die moderne Kognitionstheorie ${ }^{19}$ hat das persona-Konzept und ähnliche Ansätze zu einer metaphorisch-expressiven Theorie der Analyse ausgebaut, zumindest auf die Affinität zu älteren Konzepten hingewiesen und kategoriale Differenzierungen der möglichen Erkenntnisse vorgenommen. Dennoch bleiben kognitivistisch angelegte Analysen großenteils einer dramatischen Hermeneutik verpflichtet, indem sie die zugrunde liegenden Bewegungs- und Prozessmetaphern in den Dienst einer zu analysierenden Gestik stellen. Auch wenn damit eher eine Tiefenstruktur als eine Dramaturgie gemeint ist, ist es kein Zufall, dass Werke wie die Symphonie fantastique, Liszts Sinfonische Dichtungen, Schostakowitschs 10. Sinfonie ${ }^{20}$ und natürlich Beethovens 5. Sinfonie zu den geeigneteren Analysestücken gehören.

Aber auch der Kognitionstheorie fern stehende Ansätze und Analysen, die mit «Keimzellen`, ’kontrastierender Ableitung und sentwickelnder Variation` arbeiten, legen nicht immer offen, ob ein rezipierter Prozess als Dynamik des Werks oder als ein idealisierter Kompositionsprozess - mit dem Komponisten als dem ersten Hörer - Thema der Analyse ist ${ }^{21}$, so dass es in der inzwischen selbstverständlichen Anwendung solcher zunächst nicht metaphorisch verstandener Analyse-Modelle durchaus zu Vor-Einstellungen kommen kann, die der Nachfrage wert sind:

- Chronologie: Müssen `Erfindungskerne oder überhaupt erklingen?

- Überbietungsästhetik: Ist eine gelungene Entwicklungslogik mit der Vorstellung von Steigerung und Wachstum verbunden?

- Hörer und Komponist: Ist die `organische Formı, auch wenn sie von Komponisten auf ihre eigene Arbeit angewendet wird, ein Modell für das Produzieren oder für das Rezipieren?

\section{Keimzelle - Lobes sAusspinnungsmaximen und reduziertes Genie}

Lobe eröffnet seinen Lehrgang mit einem Probestück, welches klingend demonstrieren soll, wie aus der »Ausspinnung "22 $^{22}$ eines einzigen zweitaktigen Motivs bereits ein ausdrucksstarkes Tonstück von der Länge einer achttaktigen Periode geschaffen werden

19 Lakoff/Johnson 1997.

$20 \mathrm{Karl} /$ Robinson 1997.

21 Zum Problem der Beschreibung von Prozessen in der Schwebe zwischen Komposition, Aufführung und (Hör)analyse vgl. Dahlhaus 1987, 149 f.: »Der Disput über Wahrnehmbarkeit [...] hat die Frage nach der Struktur des als Vorgang und Vollzug begriffenen musikalischen Hörens in den Hintergrund gedrängt. Daß musikalische Form einen Prozeß darstellt, ist ein Gemeinplatz, den niemand leugnet. Es scheint aber, als seien einige Konsequenzen, die aus dem Prozeßcharakter resultieren, in der Theorie und Praxis der Analyse nicht gezogen worden, weil man sich Musik als Verlauf vorstellte, der auf ein Ergebnis, die vollendete Anschauung des Werkes, zielt. Was im Notentext räumlich vorgezeichnet ist, erwächst aus der zeitlichen Erstreckung der musikalischen Aufführung gleichsam zu einer ızweiten Räumlichkeitı: Die musikalische Phantasie erfaßt das tönende Ganze, nachdem es sukzessiv auseinandergelegt worden ist, in einer Art imaginärer Simultaneität, in der die Musik als Form zu sich selbst kommt."

22 Lobe 1858, VI. 
kann, auch wenn der Schüler neben der Kenntnis der harmonischen Hauptdreiklänge keinerlei Vorkenntnisse hat.

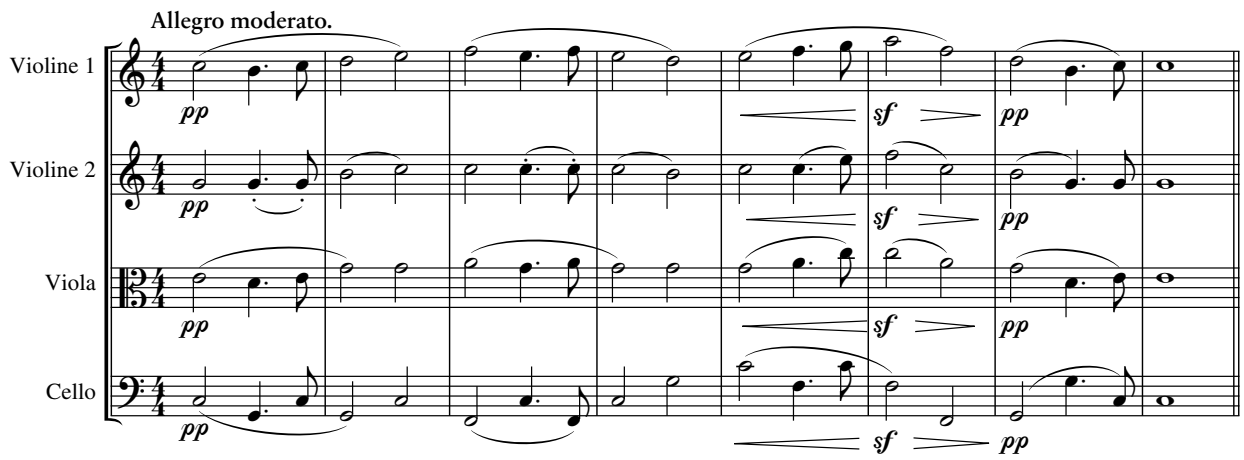

Beispiel 1: Johann Christian Lobe, Exempel aus der Einleitung der Kompositionslehre ${ }^{23}$

Die Maximen der Ausspinnung ${ }^{24}$ sind ein tragendes Konzept des Lehrgangs und begleiten fast jeden kreativen Schritt. Das Verhältnis des Ganzen zu den Teilen beschreibt Lobe folgendermaßen:

Wir haben die eintaktigen Tonbilderchen dadurch zu zweitaktigen erweitert, dass wir den ersten Takt, das erste Motiv, als Modell zu einem zweiten nahmen, dass wir den Figureninhalt des ersten Taktes im zweiten auf ganz gleiche oder doch ähnliche Weise wiederholten, dass wie entweder eine strenge oder freie Sequenz bildeten. Der Schüler fasse diese Auffassungsmaxime scharf auf, denn mit ihr führen wir ihn stufenweise und leicht bis zur Komposition der größten Tonstücke..$^{25}$

Und abschließend:

Aus geringen Keimen, aus Motivgliedern, Motiven, Abschnitten, höchstens Sätzen, welche die Modelle abgeben, werden, wie das Buch gezeigt hat, die Perioden fort- und ausgesponnen. Aus mehreren Perioden bilden sich Gruppen, aus mehreren Gruppen Theile, aus zwei oder drei Theilen entstehen ganze Formen. ${ }^{26}$

Es ist nicht neu, den Zusammenhang, die Einheit in der Mannigfaltigkeit, aus einem solchen ableitenden Verhältnis von Mikro- und Makrostruktur zu begründen. Mit den entsprechenden Größeneinheiten nimmt dies bereits Koch vor. ${ }^{27}$ Direkte Vorgänger von Lobe, wie z. B. Gottfried Weber, gehen in einer solchen Vorausnahme von Theorien der Wagner-Zeit durchaus noch weiter. Bezeichnend ist jedoch, dass Lobes anfänglich

23 Ebd.

24 Ebd., 19.

25 Ebd.

26 Ebd., 450.

27 Koch 1793, 60. 
präsentierter Tonsatz einerseits vollkommen Hugo Riemanns idealtypischer Periode ${ }^{28}$ entspricht und andererseits, bei aller oberflächlichen Ähnlichkeit mit Vorbildern aus Beethovens und Mozarts Streichquartetten, den hehren Anspruch, den ersten Zweitakter im Sinne einer Keimzelle zu entwickeln, ganz und gar nicht erfüllt. Dabei sollte man dies am wenigsten dem Verlauf der ersten Violine anlasten, die fast allein dafür verantwortlich zu sein scheint, dass diese Periode überhaupt klassische Maßstäbe erfüllt, vor allem durch den stiltypischen Wiederaufgriff des Motivs auf der dritten Tonleiterstufe im Periodennachsatz. Die konsequente Isolation der Oberstimme vom Tonsatz der übrigen drei Stimmen durch rigide Beschränkung des `Begleitsatzes` auf Dreiklänge der I., IV. und V. Stufe in Grundstellung, was nicht nur die Cellostimme, sondern mehr noch den Verlauf der zweiten Violine in Mitleidenschaft zieht und sämtliche harmonischen Zwischentöne vermeidet, zeigt die Probleme dieses didaktisch reduzierenden Ansatzes, der zu wenig zwischen einem ästhetisch hoch komplexen Postulat und seiner handwerklichen Umsetzung zu vermitteln vermag. Es zeichnet sich auch unzweifelhaft in den folgenden Übungen und Figurationsaufgaben ab, dass Lobe für die Gestaltungsaufgaben größtenteils die Rhythmik instrumentalisiert, sowie jegliche `Ausarbeitung` nicht wirklich aus der vorgegebenen Keimzelle heraus entwickelt, sondern die Gerüstsätze nackter Jelensperger-Kadenzen ${ }^{29}$ in nicht sehr reflektierter Imitation Beethoven'scher Kadenzen ornamentiert. Obwohl Lobe sich in den abschließenden Aphorismen wesentlich differenzierter äußert und zudem vereinzelt auch komplexere Stilübungen anführt, erfasst der Lehrgang in seiner Gesamtheit leider nicht mehr als die Vermittlung zwischen zweitaktigen Erfindungskernen und achttaktigen Einheiten durch vor allem rhythmische Bezüge.

Es ist bekannt, dass durch den Einfluss der Genieästhetik auch in den kompositorischen Lehrwerken große Teile der Handwerkslehre entweder isoliert oder durch ingeniöse Methoden sowie durch Inspiration von >Meisterwerken auch hier so und wäre nicht weiter von Relevanz, wenn Lobe nicht im selben Zuge eine formaldramaturgische Grundsatzentscheidung treffen würde, die die günstigste Platzierung thematischer Keimzellen in der Exposition betrifft:

Will man aber gleich am Anfang, mit dem ersten Gedanken etwas Bedeutendes, Inhaltsschweres ankündigen, so muss man vorher auch auf das Bestimmteste sich bewusst sein, voll und tief zu empfinden, was man ausdrücken will. Und wieder überblicke der Jünger von diesem Gesichtspunkte aus eine grosse Anzahl von Thematen verschiedener besserer und geringerer Komponisten, um an dem Eindruck auf sich selbst zu empfinden und zu erkennen, wie verschieden an Aufregungskraft sie sich erweisen, und wie die Wirkung stets in geringerem Grade aus den unbestimmteren, matteren, in höherem aus den bestimmteren und bestimmtesten fliesst.

Die Kunstjünger, welche diese Maxime stets im Auge behalten, einen Gemüthszustand nämlich zuerst in möglichster Energie in sich hervorzurufen, und dann nicht ruhen, bis sie seinen deutlichsten und schärfsten Ausdruck im Thema gewonnen, werden sie bald begreifen, welche Macht des musikalischen Ausdrucks sie überhaupt dadurch gewinnen, welchen glücklichen Einfluss ein glückliches Thema auf alle folgenden Gedanken 
des ganzen Stückes ausübt. Denn ein guter Anfang steigert die Kraft der Seele, versetzt sie in gute Stimmung, dass sie auch weiter gern ihr Bestes hergiebt. ${ }^{30}$

Eine unscharfe Überlagerung von Wirkung auf den Rezipienten und einer für den besonderen Zustand des Komponierens geforderten Verfasstheit des Gemüts bringt die fachliche wie methodische Problematik (nicht nur) Lobes auf den Punkt und rückt den Lehrgang zu weiten Teilen in die Nähe von - modern gesprochen - Fanliteratur.

Als Basis einer historisch informierten Formenlehre bergen Quellen wie diese das Risiko, die markante Rhetorik und Dramaturgie prominenter Eröffnungstypen als direktes Analogon zum Kompositionsprozess zu verstehen. Die exponierende Funktion eventueller Keimzellen wird überbewertet, und es gilt als selbstverständlich, dass Keimzellen tatsächlich erklingen und durch rhythmisch reduzierte Gerüstsätze aus dem Notentext zu exzerpieren sind.

\section{Handwerkliche Aspekte der Aemulatio: Dialektische Natur}

Im Vergleich zu verbreiteten metaphysischen Hypothesen, die Natur mit dem Naturschönen gleichsetzen, ist der Ansatz der Goethe-Zeit zuweilen dem heutigen Verständnis von Natur im musikalischen Kontext recht nahe und entspricht dem, was durch Tradition und Gewohnheit als natürlich empfunden wird. ${ }^{31}$ Gleichzeitig existiert ein Problembewusstsein dafür, welche Konsequenzen natura naturans als überbietendes Prinzip auf das Komponieren haben kann. ${ }^{32}$ Dass gleichzeitig durchaus der Natur nachempfundene prozessuale Dynamiken die Themen- und Motivbildung beeinflussen, macht es nicht unbedingt einfacher, die Kategorien sauber zu trennen.

Im Folgenden möchte ich exemplarische Fälle vorstellen, in denen mit den Mitteln kontrapunktischer Projektion ein naives Verständnis von Natur überboten wird, indem in traditionell gewachsene und durchaus natürlich-organischen Prozessen ähnliche Vorlagen ganz und gar unnatürliche Konstruktionsprinzipien in Form perfekter Symmetrien eingearbeitet werden und somit aus der potentiellen Keimzelle eine Problemstellung machen. Jene Problemstellung kann möglicherweise die Nachahmung eines Wachstumsprozesses an der Oberfläche sein, auf einer tieferen Ebene der formalen Konstruktion lässt sie jedoch eine andere, sehr konsequente und nicht weniger narrative Entwicklung erkennen. ${ }^{33}$

30 Lobe 1858, $437 \mathrm{f}$.

31 Ebd., 446f., wie wenig später Hanslick 1891, 152.

32 Dahlhaus 1988, 461: „Die [natürlich-entwickelnden] Prämissen sind jedoch, ohne daß sie haltlos wären, nicht unproblematisch, und zwar nicht allein darum, weil es im 19. Jahrhundert zu den ästhetischen Bedingungen des Kunstcharakters musikalischer Werke gehörte, daß sie - zumindest partiell - gewissermaßen sgegen die Konvention ‘ komponiert wurden.«

$33 \mathrm{Vgl}$. dazu das analoge Motiv in Kunst und Literatur des 19. Jahrhunderts: »In der Wende vom 18. zum 19. Jahrhundert beobachtet man in der deutschen Literatur das Aufblühen und die Ausbreitung einer Symbolik, in welcher Gegensatzpaare aus der Sphäre des Anorganisch-Mineralischen und des Beseelt-Organischen sowie deren Vertauschung eine wichtige Rolle spielen.«(Frank 1989, 11) 
Organische Themenbildung und anorganische Symmetrie: Fuge fis-Moll, WK I, BWV 859 Als Beispiel und mögliches Vorbild für romantische Themenbildungen und Formprozesse, in denen organisches Wachstum eher Konflikt als gestischer Ausdruck ist, soll zunächst das Thema der Fuge in fis-Moll BWV 859 dienen.

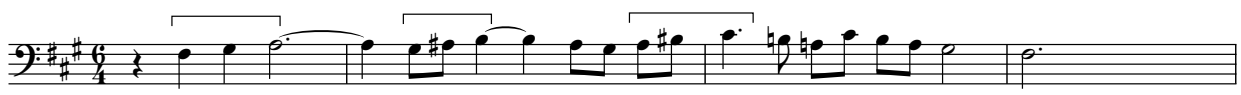

Beispiel 2: J.S. Bach, Fuge fis-Moll, BWV 859, Thema

Prozesshaft und im engeren Sinne Natur imitierend sind die rhythmische Beschleunigung des sequenzierten Eröffnungsmotivs, die kunstvolle Asymmetrie des Rhythmus und die verschobenen Taktschwerpunkte, die in großer Verlangsamung im Sinne des alten Sesquialtera-Verhältnisses zwischen einem 6/4-Metrum (1. Takt) und einem 3/2-Metrum (3. Takt) alternieren, wobei der zweite Takt zwischen den Alternativen vermittelt. Naturhaft im Sinne von gelebter Tradition ist der Thementypus, der in rhythmisch weniger komplexer Form Thema von Schulfugen ist:

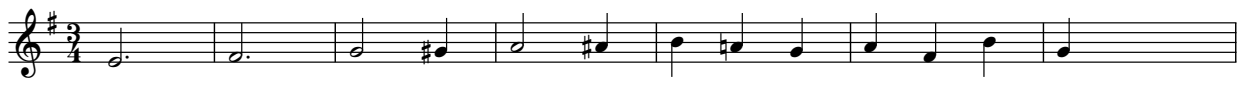

Beispiel 3: J. S. Bach, Fuge e-Moll BWV 555, Thema

Eine weitere snatürliche`Verankerung in der geistlichen Vokalmusik liegt in der großen Nähe zum Choral Ach wie flüchtig, ach wie nichtig vor, der - mehr noch als die Schulfuge - den Quintaufgang in ein sequenziertes Terzmotiv teilt.

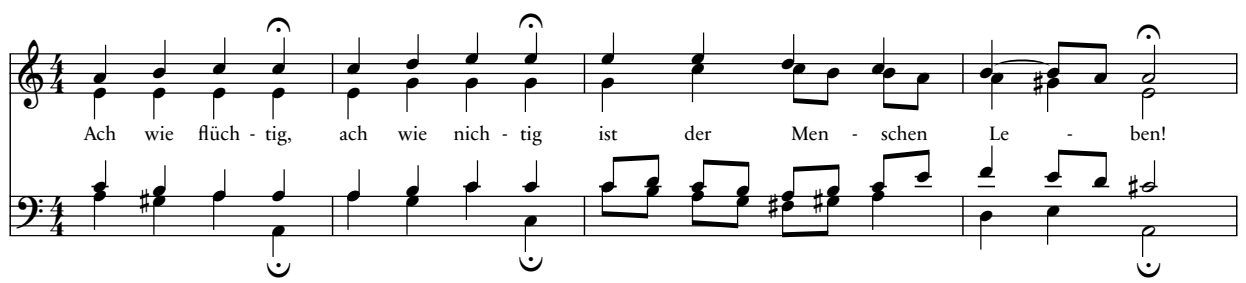

Beispiel 4: J. S. Bach Ach wie flüchtig, ach wie nichtig, Choral der Kantate BWV 26, T. 1-4

Die ınatürliche`, prozesshaft-fortspinnende Themengestaltung in BWV 859, die traditionell-natürlichen Strukturen der Tonart und die Choral-Intonation werden durch die Symmetrie einer realen Sequenz des Anfangsmotivs angestrengt: fis-gis-a, gis-ais- $h$, aishis-cis, so dass die reale Sequenz aus der Tonart heraustritt. Es ginge an diesem Ort zu weit, im Einzelnen auszuführen, was aus dieser Disposition für den Verlauf der Fuge gewonnen wird. Doch der Konflikt zwischen Symmetrie und natürlicher Harmonisierung der Tonleiter spielt weiterhin die Rolle einer Keimzelle, und als formal-gliedernder Faktor 
wird der Umstand relevant, dass das motivische Modell, der Terzanstieg re-mi-fa, sofort im ersten Kontrasubjekt gespiegelt wird (T. 4-5) und somit nicht nur `Vorahnung ‘ür die hochindividuellen Harmonisierungen dieses Themenausschnitts wird, sondern auch für die bis auf einen einzigen Ton reale Umkehrung, die in der numerischen Mitte der Fuge den zweiten Abschnitt der Großform eröffnet:

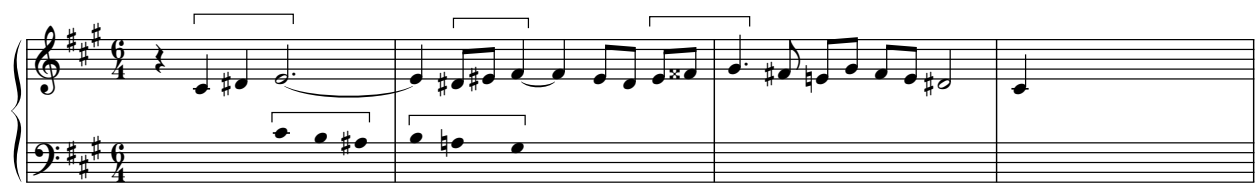

Beispiel 5: J. S. Bach, Fuge fis-Moll, BWV 859, `Ahnung، der Umkehrung, T. 4-7

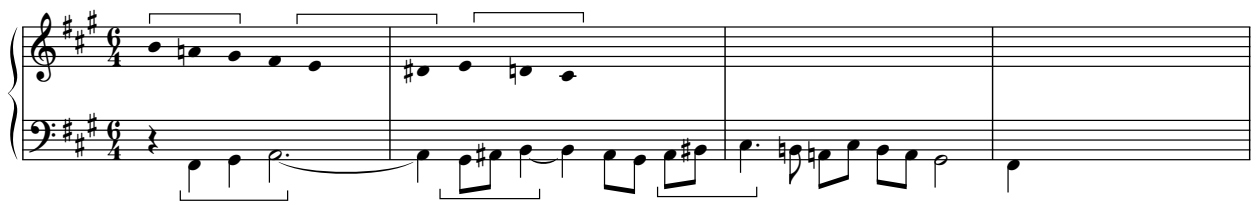

Beispiel 6: J.S. Bach, Fuge fis-Moll, BWV 859, Erweiterung der hörbaren Symmetrien von T. $4-7$ in T. 8-11

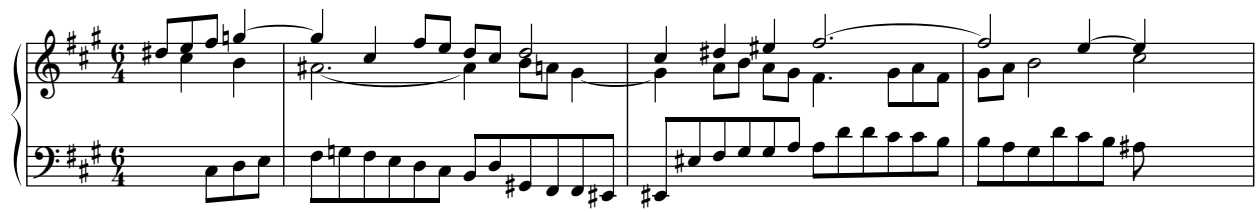

Beispiel 7: J. S. Bach, Fuge fis-Moll, BWV 859, Umkehrung in T. 18-21

Es ist diese Hybridität kontrapunktischer Optionen, die einer formalen Arbeit mit «Keimzellen` die Möglichkeit eröffnet, im Sinne eines Über-Organismus musikalische Gedanken zu formulieren: Es wird nicht nur aus einer Keimzelle abgeleitet, sondern die motivische Konstellation ist mit einer >Unruh versehen, die sich entweder in der motivischen Keimzelle selbst oder in den erst in der Entwicklung hervortretenden kontrapunktischen Optionen äußert. Entgegen des nicht nur in Analysen des 19. Jahrhunderts vorherrschenden Missverständnisses, die Keimzelle des Werkes müsse tatsächlich erklingen, und zwar exponiert zu dessen Beginn, scheint es angemessener, davon auszugehen, dass eine Keimzelle nichts ist, das überhaupt erklingen muss. Demnach sollte mit mehr Bedacht als üblich von smotivischen Erfindungskernen sesprochen werden. Auch in der Fuge fis-Moll BWV 859 ist ja nicht das Thema die Keimzelle, sondern eine prägnante Ausarbeitung, die es dem Hörer ermöglicht, die zu entwickelnde Problematik in jedem Moment der Form zu verfolgen. 
Es sollen nun vergleichbare `Erfindungskerne`, in denen organisch anmutende Entwicklungsfähigkeit und anorganisch perfekte Symmetrie eine spannungsvolle Synthese eingehen, aus dem romantischen Repertoire untersucht werden.

\section{Kontrapunkt als Formbedingung von Prozessen: Analyse}

\section{Punktualisierung durch Spiegelsymmetrie: Robert Schumann, Ein Choral}

Mit der vierstimmigen Klavierfassung des Chorals Freu dich sehr, o meine Seele, dem vierten Stück aus Album für die Jugend op. 68 treibt Schumann im schlichten Note-gegen-Note-Satz die ästhetischen Forderungen eines reinen Choralstils, wie sie in der ersten Hälfte des 19. Jahrhunderts verbreitet waren, auf die Spitze. Es ist nicht allein didaktische Reduktion, die nicht den komplexen Kantaten-Choral Bachs, sondern vor allem die Choral-Lehre $^{34}$ zur Vorlage für die Faktur des Charakterstücks macht: im 2/2-Takt, ohne Figurationen und textlos. ${ }^{35}$ Dass tatsächlich doch ein Bach'sches Vorbild im Hintergrund der reduzierenden Ableitung steht und nicht die schlichteren Choräle Grauns oder Telemanns, liegt auf Grund nicht selbstverständlicher Ähnlichkeiten in einigen Details jedoch nahe.

Nach der kurzen Definition bei Sulzer, demzufolge der beste Choralgesang der zu sein scheint, »der am einfachsten durch kleinere diatonische Intervalle fortschreitet, und die wenigsten Dissonanzen hat [...] « ${ }^{36}$, ist für dieses Ideal gerade Freu dich sehr, o meine Seele gut gewählt; denn innerhalb der Choralzeilen schreitet die Melodie fast ausschließlich in Sekundschritten fort.

Aufmerksamkeit wecken bei Schumann die Takte 17-18, in denen die rechte Hand fast signalhaft zwischen der verminderten Quinte fis- $c$ und der reinen Quinte $g$ - $d$ pendelt und der Zeile damit im Verein mit den alternierenden Quintklängen der linken Hand einen sehr profilierten und überhaupt nicht mehr am Choral orientierten Klang verleiht (Beispiel 8). Dass diese Form der vermindert-reinen Quint-Parallelen in Bachs Choralstil bei aufsteigendem Gang von der 4. zur 5. Tonleiterstufe kein Regelverstoß ist, erklärt die Besonderheit keineswegs; denn gerade das Hin-und-Her durch die Umkehr zur verminderten Quinte zurück macht die Wirkung aus, und dies ist nicht anders als absichtsvoll und lizenziös zu verstehen.

Offensichtlich ist auch die perfekte Spiegelsymmetrie der Passage, deren horizontale Spiegelachse der G-Dur-Sextakkord zum neu erreichten Hochton $d^{2}$ in der Oberstimme ist.

Vergleicht man diese Passage mit dem mutmaßlichen Vorbild aus der Kantate BWV 32, findet sich auch dort eine perfekte, am Hochton $d^{2}$ gespiegelte Symmetrie in der Führung aller vier Stimmen, die dem ersten Eindruck nach sogar größeren kontrapunktischen Aufwand als bei Schumann erfordert (Beispiel 9).

34 Z. B. Kirnberger 1776/79, 2. Teil, $22 \mathrm{ff}$.

35 Vgl. den Kommentar zur Ausgabe und Rezeption bei Poos 1995, 18-26.

36 Sulzer 1771, I, $204 \mathrm{ff}$. 

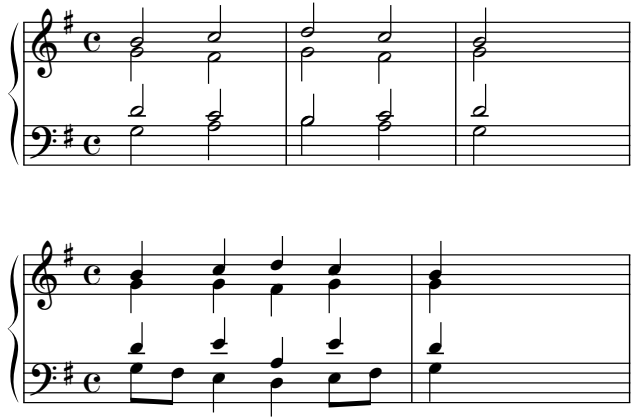

Beispiel 8: Robert Schumann, Ein Choral, T. 17-19

Beispiel 9: J. S. Bach, Choral aus BWV 32, T. 6-7 (ohne Text)

Die notwendige Bedingung der Vorlage bei Schumann, keine Figurationen zu verwenden, ist die oberflächliche Motivation für die Abweichungen vom Original. Schumann erreicht aber wesentlich mehr durch die aktualisierende Form, und zwar gerade in Hinsicht auf die Form organischer Prozesse, die als Ableitung der gesamten Form aus einem einzigen Erfindungskern heraus interpretierbar sind.

Zum einen scheint hier eines der wenigen (!) Beispiele romantischer Auseinandersetzung mit der Musik Johann Sebastian Bachs vorzuliegen, das nicht vom Schaden durch die Birnstiel-Ausgabe ${ }^{37}$ der Choralsätze, sondern offenbar von einem kreativen Gewinn zeugt: Mit der die Symmetrie überlaufenden Textverteilung »Bist du bös und ungerecht?« in der Originalzeile und ohne die Verteilung auf zwei Hände im Klavierauszug, die die Symmetrie der fünf Klänge optisch wie haptisch unterstreicht, wäre ein Großteil der Inspiration für Schumanns Charakterstück weniger aufbereitet verfügbar gewesen. Zum anderen stellt Schumanns Variante einen direkten Zusammenhang zum Stimmführungstopos der Eröffnungszeile her, dessen entwickelnde Variation eine prozessuale Logik zwischen den Zeilen erkennen lässt, die bei Bach zwar ebenso vorhanden, aber weniger plakativ herausgearbeitet ist.

Es gehört zu den standardisierten Stimmführungsmodellen im vierstimmigen Choralsatz, die ersten drei Tonleiterstufen mit einer Kombination aus - wenn man so will - dem Krebs der Oberstimme, einem Liegeton und einer Klauselbewegung zu harmonisieren. Schumann verwendet diese Figur hier ebenso wie Bach in der Choralbearbeitung der Kantate BWV 32. Auch ist in beiden Fassungen der anschließende Tonleiterausschnitt a-g-fis als Ableitung der Eröffnungsformel interpretierbar, bei Schumann allerdings lässt sich dies überdeutlich ablesen (Beispiel 10).

Dementsprechend lässt sich auch der Gang zum endgültigen hexachordalen Hochton $e^{2}$ als Ziel der Entwicklung verstehen (T. 22) und der ab Takt 25 eingeführte Durchgangston $c$ ist in diesem Falle keine Figuration, sondern der Beginn einer beschleunigten und gedrängten Rekapitulation der Takte 17-24. Es spricht für die hybride Anlage der hier realisierten sentwickelnden Variation`, dass in einem Stück, dessen satztechnische Oberfläche kaum statischer sein könnte, eine derart konzise und steigernde Entwicklung 


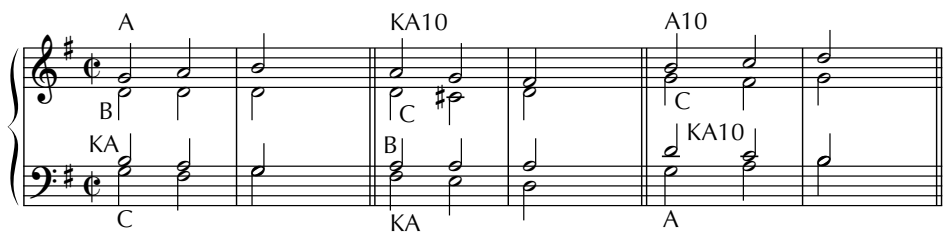

Beispiel 10: Prozess der sentwickelnden Variation` des Eröffnungsmotivs in Schumanns Ein Choral

$\mathrm{A}=$ Tonleiterausschnitt als Keimzelle, $\mathrm{B}=$ Liegeton, $\mathrm{C}=$ Klausel,

$\mathrm{KA}=$ Krebs von $\mathrm{A}$,

KA10 $=$ Krebs von A im doppelten Kontrapunkt der Dezime,

A10 $=$ Keimzelle im doppelten Kontrapunkt der Dezime

stattfindet, und zwar - ganz anders als in Lobes Maximen - mit einem gut platzierten Minimum rhythmischer Gestaltung.

Der zwar konventionelle, aber vignettenhafte Beginn des Kinder-Chorals wird über die Länge des gesamten Stückes entwickelt und in einem kritischen Moment (T. 18) durch die perfekte Spiegelsymmetrie auf einen Punkt zusammengezogen.

\section{Keine Entwicklung: Felix Mendelssohn Bartholdy, Streichquartett e-Moll, Op. 44/2, i}

Wie es in einem idealisierten Choralidiom schon eine Auffälligkeit sein kann, wenn durch das Ebenmaß hindurch eine prozesshafte Steigerung stattfindet, so bedeutet es in einer Sonatenhauptsatzform, die von konventionell drängender Motivik getragen zu sein scheint, hingegen eine Irritation, wenn die prozesshafte Entwicklung einer Keimzelle an Hand vielfältigster Ableitungen zwar angelegt ist, aber blockiert wird bzw. mit einer gewissen >Intentionslosigkeit» nicht stattfindet.

\section{Arabeske}

Zu dem hier zu untersuchenden Phänomen einer prozesshaften, natürlich-organischen Motivik $^{38}$, deren Verhalten in der Form durch perfekte, quasi an-organische Symmetrien und kontrapunktische Projektionen unterlaufen wird, gibt es bekanntlich analoge Tendenzen in bildender Kunst und Literatur des frühen 19. Jahrhunderts. Besonders im bildnerischen Denken Philipp Otto Runges gewinnt die Symmetrie der Linie über die Charakteristik des Darzustellenden hinaus an Eigenständigkeit, wobei dies - wie in der

38 Der Bezug zwischen `organischer Form ‘ und ısinfonischer Form` bzw. ıSonatenform`, der in der Regel organische Form als natürlichen Zustand sinfonischer Form voraussetzt, öffnet ein Feld, das fast so weit ist wie das des Werkbegriffs selbst, so dass es kaum möglich (oder nötig) ist, dies in Zitaten zu belegen. Einschlägig dürfte die knappe Bemerkung Schönbergs sein: »Dramatische Musik ähnelt in ihrem modulatorischen Charakter der Durchführung einer Symphonie, einer Sonate oder einer anderen geschlossenen Form.« (1992, 63) Hermann Danuser beschreibt dieses Verhältnis ähnlich knapp als Logik des »Sonatendenkens«. $(2014,312)$ Oder in größtmöglicher Allgemeinheit bei LéviStrauss: „Vor ihrer musikalischen Genese existierten die Form `Fuge` und die Form `Sonate` bereits in den Mythen.« (1989, 256f.). 
Musik - in Form von Spiegelungen oder `Echos` durch sich selbst wiederholende Linienzüge erfolgt. Obwohl es durchaus in Mendelssohns Umfeld biographische Bezüge zu Runge gab, soll es hier weniger um den Einfluss Runges auf Mendelssohn gehen, als vielmehr darum, dass ein thematischer Gedanke, der eine charakteristische Geste und damit meist einen asymmetrischen Richtungsimpuls zu verkörpern imstande wäre, gerade dieses unterlässt und sich stattdessen in eine arabeske Textur als Projektion einer Keimzelle, zu deren Natur Wachstum irritierenderweise nicht gehört, vervielfältigt und auflöst.

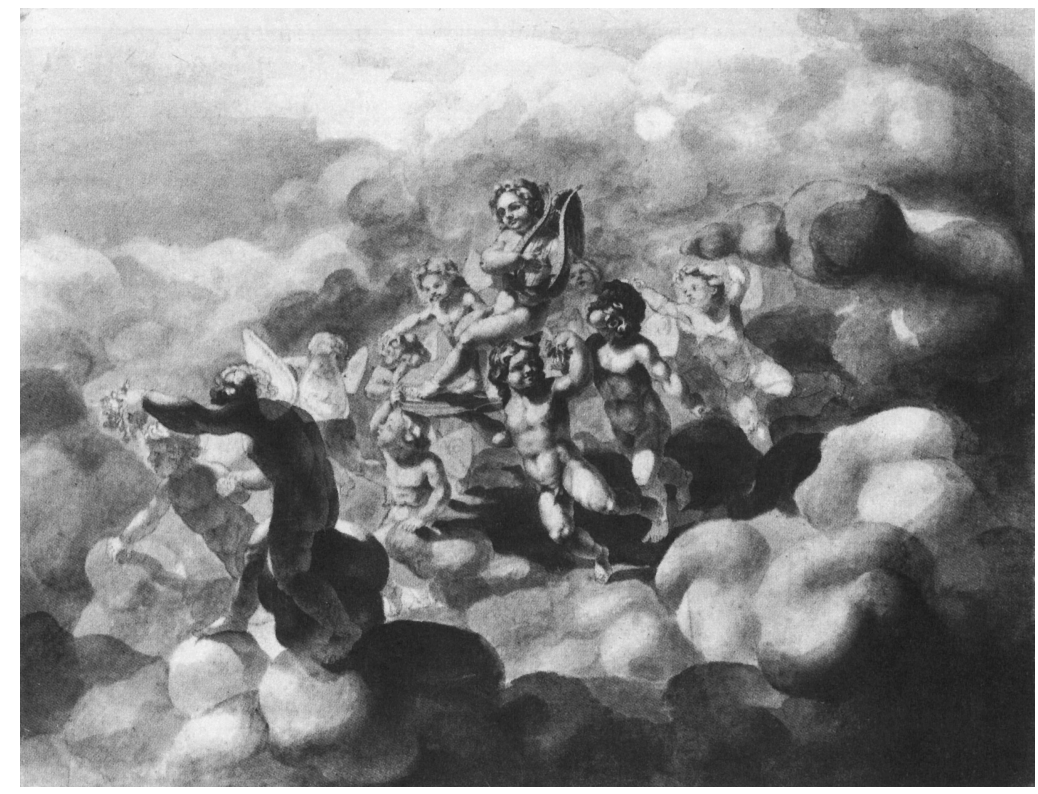

Abbildung 1: Philipp Otto Runge, Triumph des Amor ${ }^{39}$, Erste Fassung, Blei, Feder, Pinsellavierung in Grau auf weißem Papier, 31,3 x 40,4 cm, 1800, Hamburger Kunsthalle, Kupferstichkabinett

In Runges erster Fassung des Triumph des Amor ist die Figur Amors im Zentrum eines kreisenden Gewebes von ähnlichen Abbildungen seines eigenen Kinderkörpers dargestellt, wobei die Linien der Körper in elementarer Form in der Wolkenumgebung wiederkehren.

Auf der Grundlage eines musikalischen Analogons zum ınoch-nicht-ausgebildeten Körper, der Vorstellung eines ınoch-nicht-gebildeten` Themas, das die Sonatenform nicht durch die Individualität seiner Charakterbildung oder seines Typus bestimmt, sondern aus Spiegelungen und Projektionen seiner selbst entfaltet, ohne sie zur Bestimmtheit anwachsen zu lassen, sei nun ein Überblick über die Symmetrien des Kopfsatzes des e-Moll-Streichquartetts Op. 44/2 gegeben.

39 Zitiert nach Lange 2010, 174. 

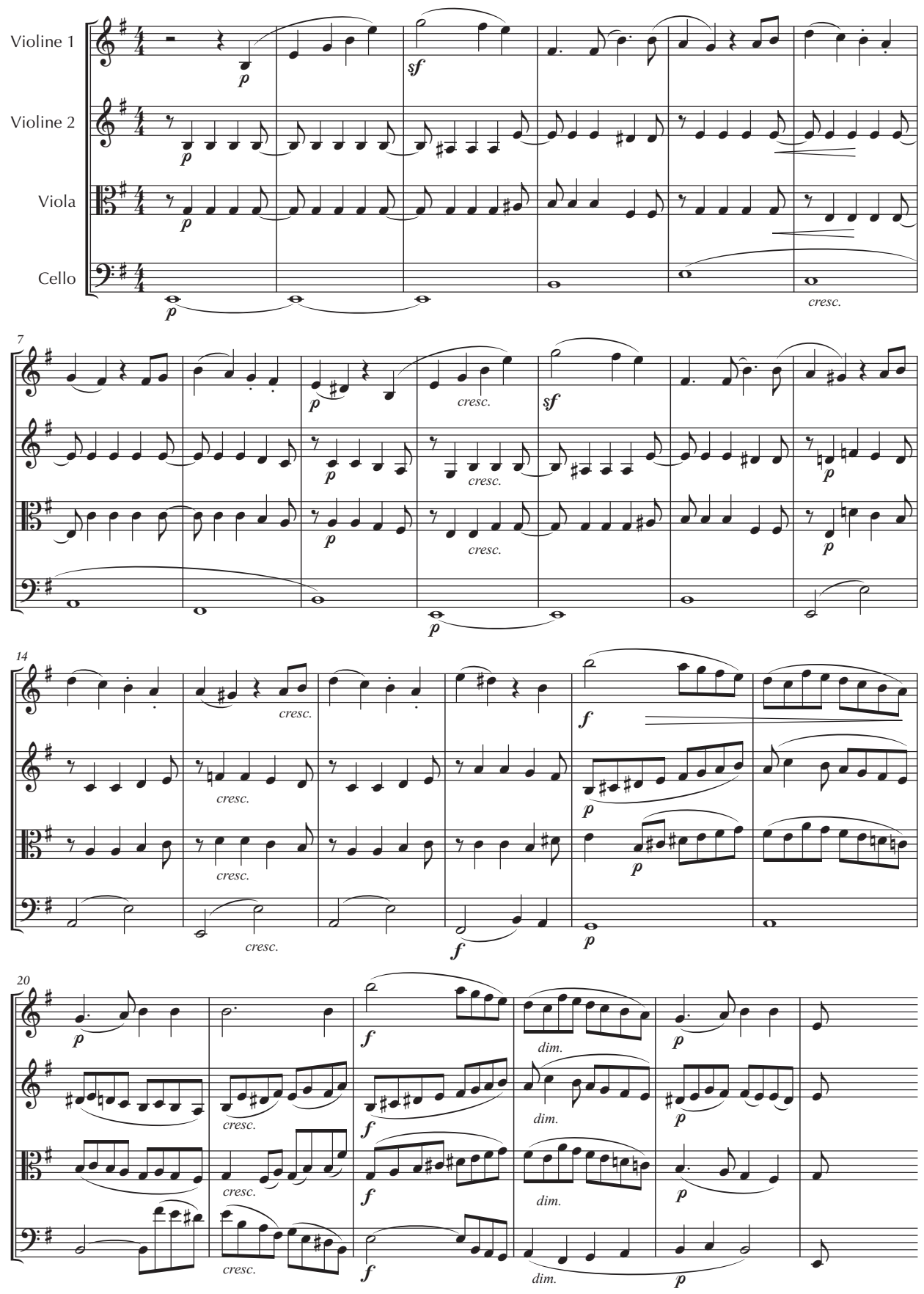

Beispiel 11: Felix Mendelssohn Bartholdy, Streichquartett e-Moll Op. 44/2, i, T. 1-25

114 | ZGMTH 12/1 (2015) 
Die sprachnahen Gesten der ersten Violine verhüllen die symmetrische Figur kaum, auf der die erste Periode des Hauptsatzes aufgebaut ist. Der Erfindungskern der Periode ist ein Melodie-Zug vom Grundton zur Terz der Ausgangstonart, der nicht weiter Aufmerksamkeit erregte, wenn er nicht auf mehreren Ebenen der Form wiederholt aufträte:

- als Umkehrung in den Takten 6-8,

- als chromatische Variante und Krise der Periode durch die Einführung von gis im Perioden-Nachsatz,

- als Umkehrung im metrisch betonten 2. Takt (= absolut 3. Takt) der Periode aus den Spitzentönen der Oberstimme.
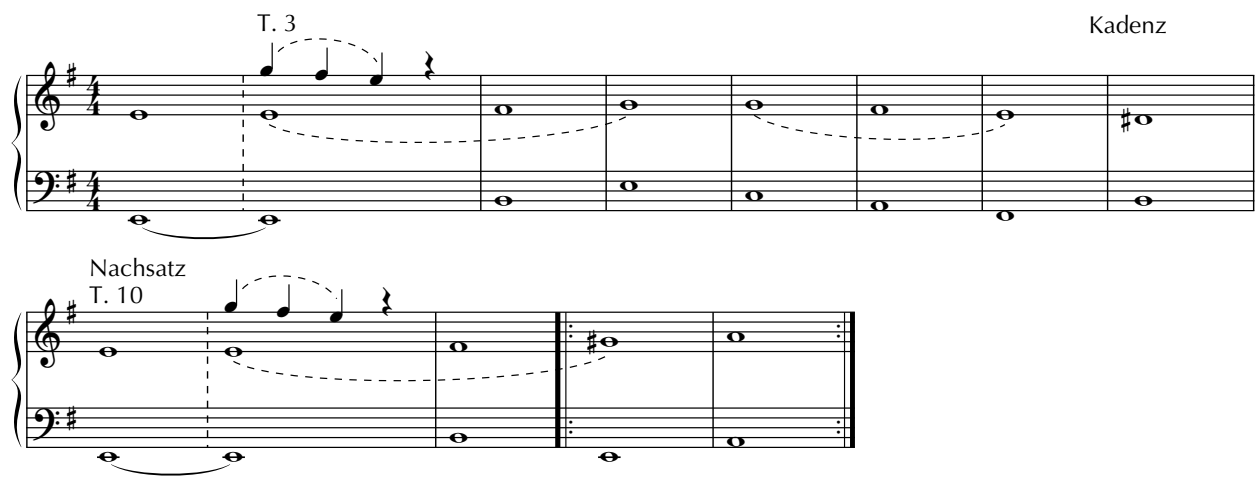

Beispiel 12: Felix Mendelssohn Bartholdy, Streichquartett e-Moll Op. 44/2, i, motivische Reduktion der eröffnenden Periode T. 2-15

Der Perioden-Nachsatz resümiert die Figur in der Schlusskadenz, er resümiert aber ebenfalls die Verdichtung des Ornaments auf drei Ebenen der Struktur:

- als Gerüst der Viertakt-Gruppe in Takt 18-21 bzw. 22-25,

- als motivischer Gegenbewegungs-Kontrapunkt zwischen Violine und Viola in Takt 18 bzw. 22,

- als ornamentale Struktur erweitert zur Gestaltung der Höhepunkte ebenda.

\section{T. $18 / 22 \mathrm{ff}$.}

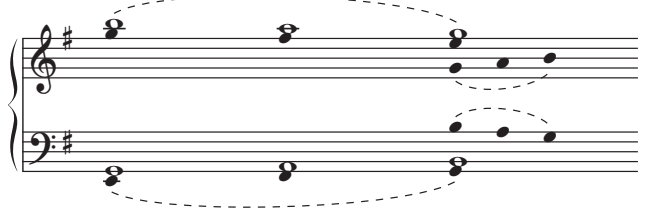

Beispiel 13: Felix Mendelssohn Bartholdy,

Streichquartett e-Moll Op. 44/2, i,

T. $18 \mathrm{ff}$. bzw. T. $22 \mathrm{ff}$., Reduktion

Erst im kleinsten Maßstab, auf der Ebene der ornamentalen Verdichtung, nimmt Mendelssohn die Paarstruktur des Dezimenkontrapunkts wahr. 


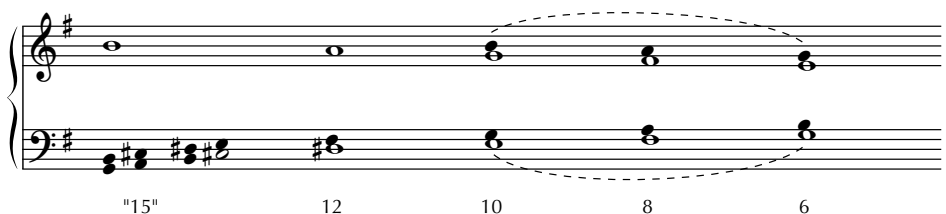

Beispiel 14: Felix Mendelssohn Bartholdy, Streichquartett e-Moll Op. 44/2, i, T. 18/22-24, Reduktion

Wie oft ist die Symmetrie der Periode des Seitensatzes um die Hälfte diminuiert und darum mehr mit dem nächstkleineren Maßstab, der Ebene der Motivik, verwoben.

Das umgekehrte und in die parallele Durtonart, also um eine Terz höher auf die Töne h-a-g versetzte Gerüst wird zwar emanzipierter behandelt, dafür werden symmetrische Bildungen auf der Ebene der Motivik umso deutlicher angespielt.

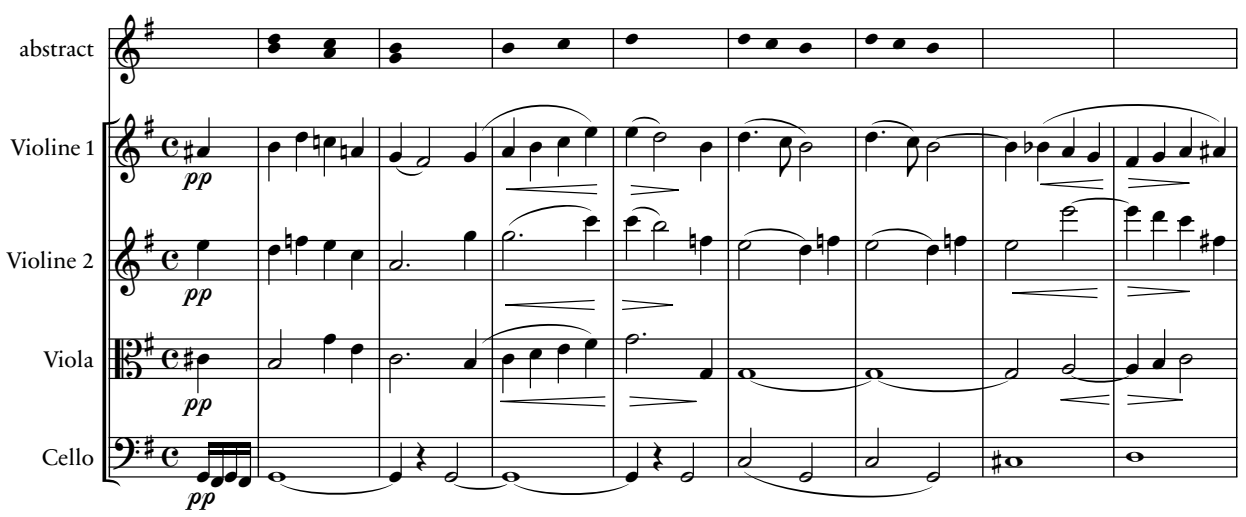

Beispiel 15: Felix Mendelssohn Bartholdy, Streichquartett e-Moll op. 44/2, i, T. 53 m.A-60, Seitensatz und Abstraktion

Der Tonartenplan der Exposition bildet die Figur e-fis-g in der formal größeren Dimension $\mathrm{ab}$, indem die Seitensatztonart G-Dur trugschlüssig über einen langen, vorausgehenden Orgelpunkt fis erreicht wird (Beispiel 16)

In der Durchführung, die nur sehr wenig moduliert, bildet sich C-Dur als Haupttonart sowohl der Rekapitulation des Haupt- als auch des Seitensatzes heraus. Mit C-Dur ist im Tonartenplan die Symmetrie vollendet als Spiegelung des Oberterzverhältnisses zwischen Haupt- und Seitensatz in der Exposition. Die Symmetrie der Figur erfasst also, ausgehend vom miniaturhaften Ornament des Dezimenkontrapunkts, auch die Großform in der Vertikalen (Beispiel 17).

Bemerkenswert sind die kommentarhaften Takte 265-268 der Coda, in denen der Hauptsatz auf diese tonale und kontrapunktische Symmetrie reduziert erklingt, im Pianissimo, als solle ein Geheimnis verraten werden, wobei die Strukturen sauber getrennt auf die Instrumente verteilt sind (Beispiel 18). 
T. $1-35$
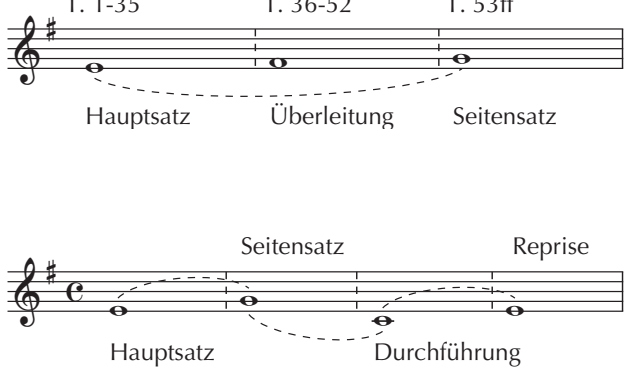

Beispiel 16: Felix Mendelssohn Bartholdy, Streichquartett e-Moll op. 44/2, i, Tonartenplan der Exposition in linearer Darstellung

Beispiel 17: Felix Mendelssohn Bartholdy, Streichquartett e-Moll op. 44/2, i, Tonartenplan des gesamten Satzes als augmentierte Keimzelle`

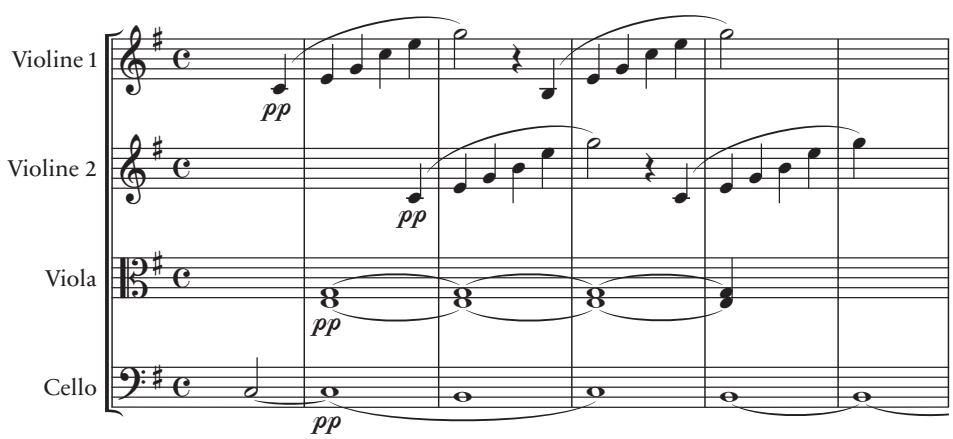

Beispiel 18: Felix Mendelssohn Bartholdy, Streichquartett e-Moll op. 44/2, i

T. 265-268

Formulierte man die Keimzelle der Symmetrie einstimmig als Tonfolge e-fis-g, schiene sie den motivischen Strukturen nahe zu stehen, die Krummacher und Dahlhaus in der Beethoven-Analyse als "Submotive ${ }^{40}$ bezeichnet haben. Auch zeigt die innere Entwicklung der figurativen Verarbeitung des Motivs im Satzverlauf sehr deutliche Züge dessen, was Schönberg an Brahms als "Motiv der Variation « ${ }^{41}$ analytisch hervorhob. Die `Zelle « e-fis-g demzufolge auch als ıSubmotiv zu bezeichnen, trifft es nur fast: Der Tonfolge kommt kein Vorrang vor ihren texturhaften Projektionen zu; denn sie existiert als Einzelidee gar nicht und kann auch nicht als Einzelidee Position eines dialektischen Prozesses sein. Auch ist der aus dieser Textur entfaltete Verlauf der Sonatenform zwar dialektisch, aber in seiner Selbstreflexion eher anschauend als produktorientiert.

Durch den Umstand, dass es sich bei der Keimzelle um dieselbe Tonhöhenstruktur wie in der fis-Moll-Fuge und im analysierten Choral handelt, wird besonders deutlich, dass es nicht auf die eigentliche Gestalt des Motivs ankommt, wenn es darum geht, eine >organische Form ‘ als steigernden Prozess, wie es dem ästhetischen Empfinden der Zeit als vollendet entsprach, oder eben weniger gestisch steigernd bzw. organisch wachsend

40 Krummacher 1980, 126-127 und Dahlhaus 1987a, $126 \mathrm{ff}$. und 245ff., beide in Weiterführung der Ansätze Rudolph Retis (1961).

41 Schönberg 1979, 77-80. 
zu inszenieren. Mendelssohn ist für diesen Verzicht auf authentisch-affirmative Steigerungen schon zu Lebzeiten immer wieder kritisiert worden, möglicherweise auch auf Grund der impliziten Ideologie romantischer Formästhetik ${ }^{42}$, die nicht in der Lage war, weniger ausgestellte kontrapunktische Ableitungen auch dann als Entwicklung oder organische Entfaltung zu erleben, wenn dies in einem übergeordneten Decrescendo oder einem Zurückhalten von Intensität stattfand. ${ }^{43}$

\section{Hexachordum fictum: Johannes Brahms, Intermezzo op. 116/5}

Symmetrie mag sich vor allem dem Spieler als übergeordnetes Motiv aufdrängen, da im fünften Intermezzo - als eine Art körperliches Gegenstück zum Intermezzo op. 116/4 anstatt der Arme nun die Hände extrem gekreuzt sind. Die Daumen der beiden Hände liegen ungewöhnlich oft weit ineinander verschränkt. Die Linearität dessen, was beim Spielen zu hören ist, entspricht keinem vertrauten Spielgefühl. Eine vergleichbare `Hemmung` ergibt sich aus der Phasenverschiebung der Zusammenklänge, welche vollgriffig auf unbetonter Zeit stehen. Das Zurückschnellen der Hände in die konventionelle Spielposition und wieder in die gekreuzte Position vor die Körpermitte stellt einen großen koordinatorischen Aufwand dar, so dass die sehr schlichte Motivik Ton für Ton erworben werden muss.

Fordernd für das harmonische Verständnis ist der Umstand, dass auf den betonten Zählzeiten zunächst nur harmoniefremde Nebennoten erklingen, und zwar in snackter`Zweistimmigkeit derart verschleiert, dass der nachvollziehende Hörer ergänzen muss, ob sich die Zweiklänge zu vollen Nebenharmonien zusammenschließen könnten (T. 1, Zz. 1) oder ob der Zweiklang rein skalar zustande kommt (T. 1, Zz. 4). Jeweils nach zwei Takten, mit dem Ende des Motivs, wird ein konsonanter Klang erreicht.

Hört man nur auf diese Spur der konsonanten Sextakkorde, ergibt sich ein schlichter Fauxbourdonsatz als Satzmodell, der die förmliche Ausweichung in die Tonart der Oberquinte vorbereitet:

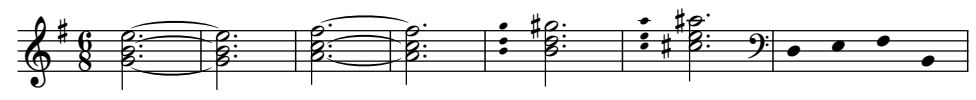

Beispiel 19: Johannes Brahms, Intermezzo op. 116/5, T. 1-12, Reduktion I

Das eröffnende Motiv der Oberstimme $h$-c kann aber aus wenigstens zwei Gründen nicht allein ornamental gehört werden, da es einerseits eine für e-Moll typische Eröff-

42 Vgl. Dahlhaus 1974, 7-9.

43 Vgl. auch die implizite Forderung nach Produktivität in Adornos hegelisch geprägter Terminologie, wenn Termini wie `Werden`, ’Kraftzentren` und `Totalitätı, zwar anti-romantisch, aber unmissverständlich einen im herkömmlich-naturalistischen Sinne ergebnisorientierten Herstellungsprozess beschreiben: „Was irgend am Kunstwerk Totalität heißen darf, ist nicht das all seine Teile integrierende Gefüge. Es bleibt auch in seiner Objektivation ein vermöge der in ihm wirksamen Tendenzen erst sich Herstellendes. Umgekehrt sind die Teile nicht, als was sie durch Analyse fast unvermeidlich verkannt werden, Gegebenheiten: eher Kraftzentren, die zum Ganzen treiben.« (1970, 266) 
nung ist und zum anderen deutlich mit dem Beginn des vorausgehenden Intermezzos in E-Dur korrespondiert.
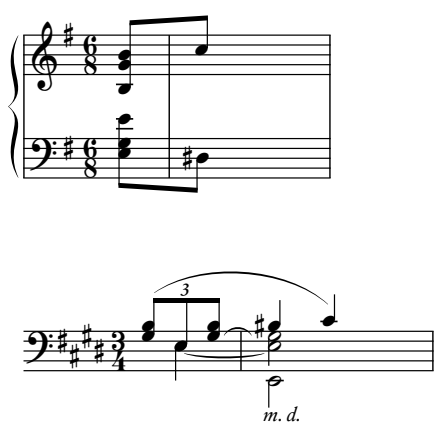

Beispiel 20: Johannes Brahms, Intermezzo op. 116/5, Eröffnungsmotiv

Beispiel 21: Johannes Brahms, Intermezzo op. 116/4, Eröffnungsmotiv

Insofern ergäbe sich ein zweiter, alternativer Gerüstsatz, der tendenziell eher auf den Taktanfängen zu hören ist.

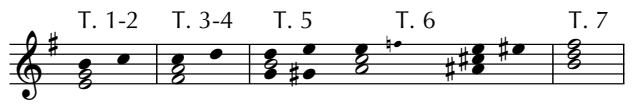

Beispiel 22: Johannes Brahms, Intermezzo op. 116/5, T. 1-7, Reduktion II

Die dieser Variante zugrundeliegende 5-6-Konsekutive eröffnet auch den Kontrastteil des Intermezzos ab den Takten 11-12, die ebenso wenig ornamental gehört werden kann, auch wenn Brahms die Sequenz hier sehr provokant mit gleich drei verminderten Dreiklängen in Grundstellung inszeniert und es wegen der gespiegelten Stimmführung im Bass nie zu der glücklichen Auflösung in die Sexte kommen lässt, die eine konventionelle Form böte.

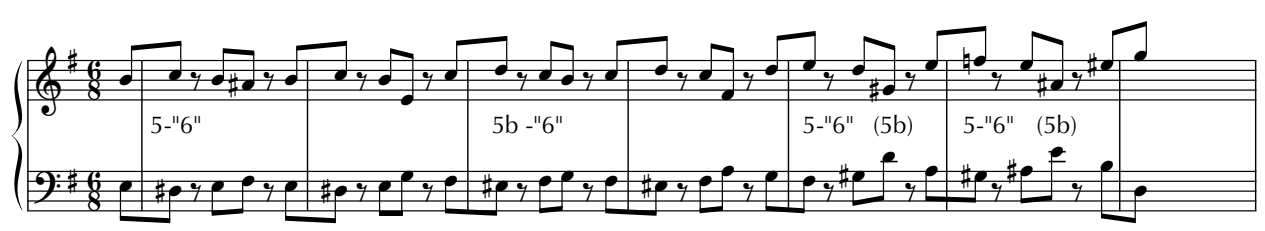

Beispiel 23: Johannes Brahms, Intermezzo, op. 116/5, T. 1-7, Sequenzstruktur der Mittelstimmen

Da das Motiv der ersten beiden Takte offensichtlich zur Ableitung des gesamten 'Satzes ${ }^{44}$ des A-Teils der vorliegenden dreiteiligen Liedform als Modell dient und auch im Kontrastteil - nach E-Dur transformiert - beibehalten wird, spricht zunächst einiges dafür, dieses Motiv ganz herkömmlich als `Keimzelle` oder `Erfindungskern` zu interpretieren. Neben

44 Auch wenn offensichtlich ist, dass die sich in Ratz' Terminologie verkörpernde Idee hier durch die scheinbar wenig empathische Mechanik der Faktur bewusst unterlaufen wird, ist der Begriff ,Satz' als traditionelle Folie sinnvoll. S. Ratz 1973, 26 ff. 
den bereits genannten Optionen Griffsymmetrie, Spiegelsymmetrie des zweistimmigen Satzes in den Mittelstimmen, 5-6-Konsekutive und Fauxbourdonsatz entstehen weitere Optionen für fortzusetzende ‘Keime`, auch wenn diese nun der Griffstruktur zuwiderlaufen: Im Abstand einer punktierten Viertelnote, also auf der Ebene der >Prolatio々, findet ein Unterquintkanon zwischen den Hauptstimmen statt ${ }^{45}$, wobei dem Dux in der rechten Hand jeweils eine eingerichtete Version in der linken Hand folgt. Innerhalb der modellhaften Setzung in den Takten 1-2 ist die Einrichtung sogar konventionell nach der Mechanik einer sogenannten stonalen Beantwortung`.

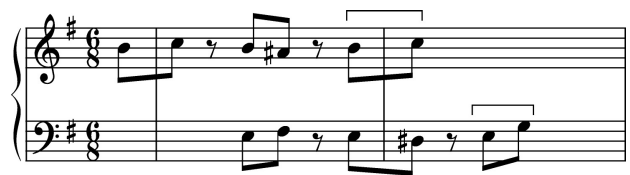

Beispiel 24: Johannes Brahms, Intermezzo, op. 116/5, Sequenz der Mittelstimmen als eingerichteter Unterquintkanon

Da auch die h-Moll-Kadenz durch einen Umkehrungskanon im Abstand einer punktierten Viertel überlagert ist, was wesentlich zu ihrer Dissonanz beiträgt, ist es angebracht, den Kanon sad minimamı zur Disposition der Keimzelle hinzuzunehmen.

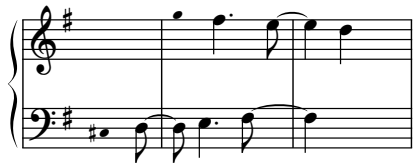

Beispiel 25: Johannes Brahms, Intermezzo op. 116/5, T. 6-8 als Umkehrungskanon, Reduktion

Es ist an diesem Punkt der Analyse angebracht, zu der Frage zurückzukommen, inwieweit eine formale Dramaturgie, die offenbar in der Exposition Einblicke in strukturelle Prinzipien gibt, und zwar in einen motivischen Erfindungskern gedrängt, dessen Entwicklung sich verfolgen lässt, als deckungsgleich angenommen werden sollte mit der Rekonstruktion von Kompositionsprinzipien, zumal die Idee einer eröffnend exponierten `Keimzelle` oft, wie auch hier, zunächst plausible Einsichten zutage fördert. Allerdings - und das erhält die Skepsis an der Möglichkeit solcher >Einblicke in die Werkstattı am Leben - ist es schwer vorzustellen, dass ein solcher Prozess nicht unterlaufen wird, gerade weil er zu erwarten ist.

Zu Beginn des Mittelteils kommt es in Takt 14 zur Dissonanz der übermäßigen Oktave. Diese denkbar scharfe Dissonanz ist weder formale Krisis einer Steigerung noch irgendwie eine Art Ende eines Prozesses. Im Gegenteil: Die formale Position ist so unauffällig, dass die Stelle wie ein Fehler klänge, wäre sie nicht motivisch und harmonisch unvermeidbar. Ähnlich wie die Fesselung der Hände im Eröffnungsmotiv 'geht es hier einfach nicht anders`. Dass die Passage intentional markiert ist, wird durch die vom Vorigen abgesetzte Lage im tieferen Register deutlich (Beispiel 26).

Untersucht man die Passage weiterhin svon vorn ‘, d. h. als Station auf einer Entwicklung aus der eingangs exponierten Keimzelle, so finden sich weitgehend dieselben Koordinaten (Beispiel 27):

45 Zum Kanon bei Brahms als formgestaltendes Prinzip vgl. Ickstadt 2014. 
- der Unterquintkanon im Abstand einer punktierten Viertel,

- die 5-6-Konsekutive, allerdings auf das doppelte Tempo beschleunigt und mit einer zusätzlichen 7-6-Vorhaltskette versehen,

- und eine wieder verlangsamte 5-6-Konsekutive an der chromatisch erweiterten Tonleiterstufe, die eine Terz tiefer hinzutritt.

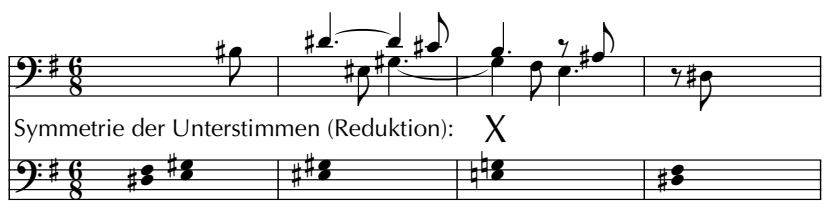

Beispiel 26: Johannes Brahms, Intermezzo op. 116/5, smarkierte Passage T. 12-15

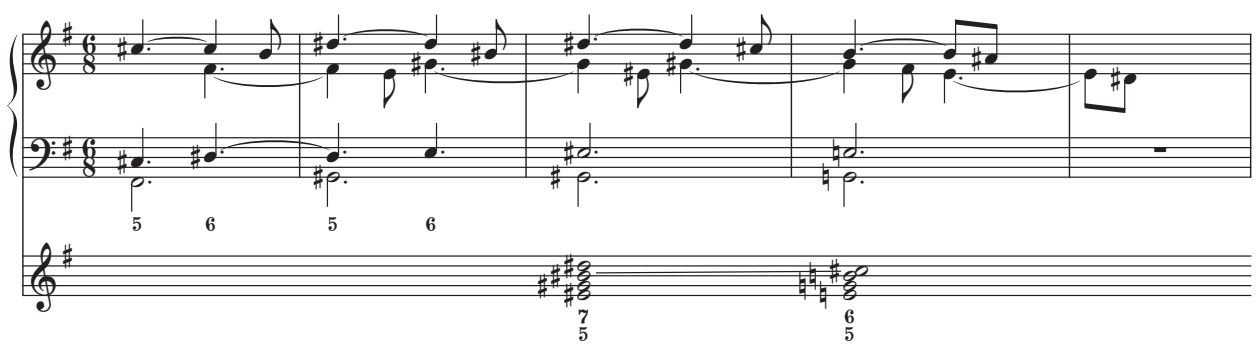

Beispiel 27: Johannes Brahms, Intermezzo op. 116/5, kanonisch-sequenzierende Strukturen T. $12-15$

Zu diesen bekannten, aus dem A-Teil entwickelten Elementen tritt mit der chromatischen Alteration eis/gis-e/g eine neue Idee hinzu, deren Herkunft und formale Verankerung sich nicht allein aus dem Beginn des Stücks und seiner Transformation von e-Moll nach E-Dur erklären lässt.

Symmetrie als formal übergeordnete Idee: potenzierte Form

Op. 116/5 beginnt mit einem Halbtonmotiv mi-fa in der Oberstimme in einem stilisierten e-Phrygisch und endet mit der sehr ausgedehnten Umkehrung des Motivs. ${ }^{46}$ Die Rückführung zur Reprise in der Unterquinte ist mit einer ebenfalls ausgedehnten phrygischen Wendung gestaltet, die dieselbe Spiegelsymmetrie aufweist (T. 20-28).

Das im ersten Takt auf der motivischen Mikroebene exponierte Motiv wird gespiegelt und formkonstituierend auf die Makroebene projiziert. Dieses Verfahren, formale Strukturen auf diese Weise fraktal anzulegen, wie es im Übrigen auch im ersten Satz von Mendelssohns op. 44/2 beobachtet werden konnte, hat Alfred Lorenz ${ }^{47}$ - angewandt

46 Vgl. Arthur Komars Konstruktion eines Schenker-Graphen für sphrygisierende` Formverläufe, der dieselbe Symmetrie am Beispiel des Liedes Ich hab' im Traum geweinet rekonstruiert $(1971,88)$.

47 Lorenz 1924. 
auf die Analyse von Wagners Musikdramen - spotenzierte Form genannt, auch hier sicherlich mit dem metaphorischen Überschuss, im Wachsen der motivisch organisierten Formteile läge ein Ansteigen von Kraft und Intensität, aber keine Verlangsamung, kein Decrescendo. Während auch Lorenz offenbar selbstverständlich von einer Kongruenz von motivischer Geste und dem, was man gemeinhin `Ausdrucksgehaltı nennt, auf der einen Seite und formaler Disposition auf der anderen Seite ausgeht, zeigen bloße Stichproben wie Mendelssohns op. 44/2 und Brahms' op. 116/5, dass eine zwar stringente, aber statische oder sogar abbauende Motivik Strukturen wachsender Formteile freilegen kann, wie sie am plakativsten in Riemanns `Taktgruppenmetrikı dokumentiert, in der Musiktheorie des 19. Jahrhunderts aber schon vorher breit angelegt sind. Die bewusste Arbeit mit einer motivischen Interaktion zwischen Mikro- und Makrostruktur ist demnach unabhängig von einer mit `Wachsen s assoziierten Dramaturgie.

Auch der in Takt 1 initiierte Unterquintkanon ist durch die Unterquint-Reprise augmentiert und schließlich zusammenfassend markiert durch den motivischen Kanon in den Takten 35-36, wo die für phrygische Wendungen typische Umspielung des Quintkanons c-h-ais, diesmal umgekehrt, von der Unterquintvariante vorimitiert wird (T. 34-35). In Analogie zu einer derartigen spiegelsymmetrischen Auskomposition des Schrittes von der 5. zur 6. Stufe, entspräche die Passage der Takte 13-15 einer durch den Kanon intensivierten Transformation desselben Motivs in eine in jedem Falle künstliche Durvariante ${ }^{48}$; denn sphrygisierende< Topoi haben kein durales Pendant.

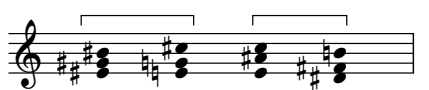

Beispiel 28: Johannes Brahms, Intermezzo op. 116/5, T. 13-15 als artifizielle Dur-Form einer phrygischen Wendung

\section{IInschrift: : Hexachordum fictum}

Die im Kontrastteil von Brahms' op. 116/5 stattfindende Überlagerung von e-Moll und E-Dur spielt nicht zuletzt mit der Enharmonik der für den Unterquintkanon der Takte 13-15 relevanten Halbtonschritte. Die scheinbare Identität zwischen his-h und dem ınatürlichen $c-h$, sowie eis-e und $f$-e ist beabsichtigt. Es deutet allerdings einiges darauf hin, dass Brahms mit dem komprimierten Portrait der Tonarten noch etwas weiter geht und noch zusätzliche kontrapunktische Chiffren als sKeimzelle` verwendet, die allerdings in den ersten beiden Takten noch nicht angedeutet sind.

Der in den Oberstimmen der Takte 13-15 erklingende Unterquintkanon ähnelt einem bekannten und mit E-Dur auch assoziierten obbligo ${ }^{49}$, nämlich ut-re-fa-mi-re-ut. ${ }^{50}$ Die in dem überlieferten Motiv verkörperten hexachordalen Strukturen sind hier eklatant falsch

48 So als Moll-Dur-Gegensatzpaare im 3. Satz von Felix Mendelssohns Schottischer Sinfonie, in Franz Schuberts Lied Trockne Blumen, im Finale von Richard Wagners Walküre sowie in den Schlusstakten des E-Dur-Präludiums aus dem ersten Band des Wohltemperierten Klaviers.

49 Zum Begriff vgl. Bornstein 2004, 151-172.

50 Vgl. Hübler 1989, der ebenfalls melodische Keimzellen, ähnlich den Pattern Rudolph Retis (1961), aus den Intermezzi herausarbeitet, allerdings wesentlich freier in der Behandlung, als es hier gemeint ist. 
im Sinne eines extremen shexachordum fictumı und korrigieren sich durch die chromatisch verfälschte Symmetrie beim Abstieg selbst.

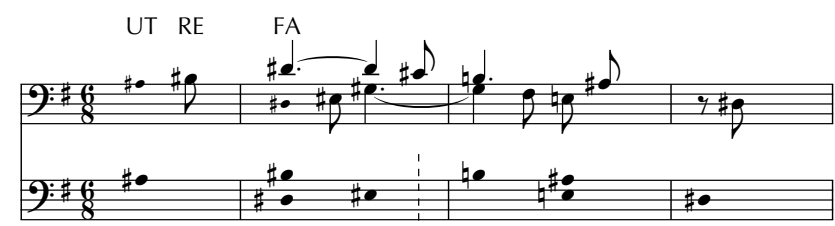

Beispiel 29: Johannes Brahms, Intermezzo op. 116/5, T. 12-15 als verschleierte Präsentation des ut-re-fa-mi-re-ut-Soggettos

Dieser verborgene cantus firmus wäre als Analysehypothese natürlich zu spekulativ, wenn die Tonfolge nicht im weiteren Verlauf zunehmend weniger enigmatisch präsentiert würde.

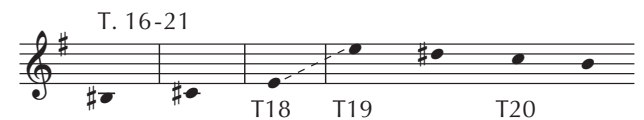

Beispiel 30: Johannes Brahms, Intermezzo op. 116/5, allmähliche sKlärung` des Soggettos in T. 16-21 (25)

Am deutlichsten, ganz im Sinne einer Auflösung, klingt das Motiv durch die Schlusskadenz hindurch:

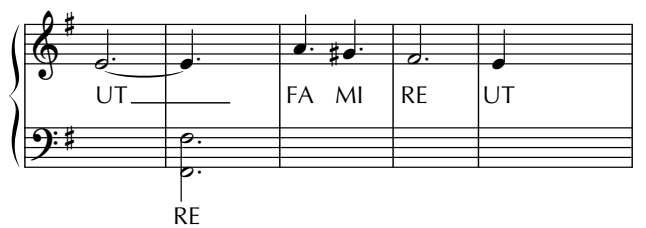

Beispiel 31: Johannes Brahms, Intermezzo op. 116/5, >Enthüllung des Motivs in T. $35-39$

Die Formel dringt nicht allein deshalb durch den Akkordsatz an die Oberfläche der Wahrnehmung, weil Brahms ihre Töne jeweils auf die in der Harmonie aktivsten Bestandteile gelegt hat:

- e als große Septime,

- fis als unerwartete chromatische Alternative von $f$ und sehr deutlich als Basston eines Terzquartakkords,

- a als einzige Veränderung zum Klang vorher,

- gis als Auflösung von a.

Danach liegt der Melodiezug in einer Stimme und müsste schon allein deswegen nicht mehr unterstützt werden, weil die Tenorklausel fis-e ohnehin erwartet wird und der Hörer die notwendige Assoziation zur bekannten Formel auch bereits vollzogen haben dürfte. 
Brahms greift mit der Konfrontation zweier hexachordal gebundener Formeln, dem ut-re-fa-mi und dem phrygischen mi-fa ein sehr altes, mit der tonalen Identität von E-Dur verknüpftes Problem auf. In beiden Bänden des Wohltemperierten Klaviers geht Bach in den E-Dur-Stücken darauf ein, wobei die E-Dur-Fuge des zweiten Bandes ein direktes Vorbild sein dürfte.

In einer Überblendung des sich in der sogenannten 'wohltemperierten Stimmung, abbildenden Ideals der sreinen Stimmung in einigen älteren Tonarten, zu denen E-Dur nicht gehört, und einem Ausspielen kirchentonaler Topoi im Kontext neuer Tonarten sind gerade der Ganztonschritt zwischen e und fis als ut und re eines shexachordum fictum auf e und die enharmonisch interessante Alternative der Ganztonschritte es- $f$ und dis-eis Elemente eines gemeinsamen Spannungsfelds. Beispiele wie das sehr dissonant auf e transponierte Thema der C-Dur-Fuge BWV 846, Takte 12-13, die phrygisierte Variante des Themas der Fuge in dis-Moll BWV 853 (T. 82) sowie die Enharmonik zwischen es-Moll-Präludium und dis-Moll-Fuge sind nur sehr plakative Momente, die auf ein Bewusstsein für die Enharmonizität von E-Dur/Fes-Dur hinweisen, sowie das Portrait gerade dieser Tonart als fiktive.

In den Metamorphosen des Fugenthemas in der zweiten Hälfte der Fuge E-Dur BWV 878 ist das Alternieren zwischen tonal richtiger phrygischer Form und tonal fiktiver Originalgestalt des Soggettos stringent entwickelt.

T. 20-22

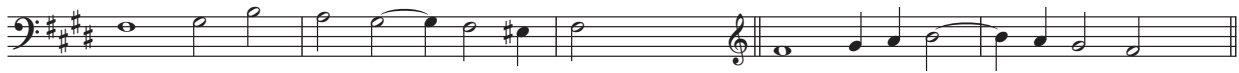

T. 25-27

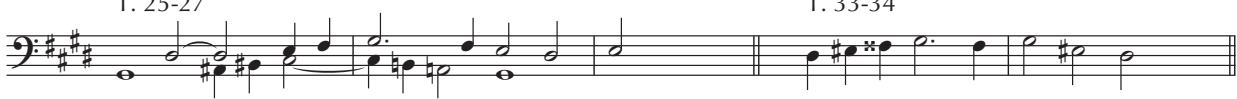

Beispiel 32: J. S. Bach, BWV 878, Metamorphose des Fugenthemas:

- T. 20-22: mollare Fassung re-mi-sol,

- T. 23-24: mollare, symmetrische Fassung mit Durchgangsnote,

- T. 25-27: phrygische Fassung im Quintkanon mit chromatischer Alternative

- T. 33-34: senharmonische Fassung,

Besonders die Themenvarianten der Takte 25-27 und 33-34 legen nahe, dass die kontrapunktischen Implikationen in Brahms' op. 116/5 von der Fuge BWV 878 inspiriert gewesen sein dürften. Aber allein schon, dass es sich mit dem Soggetto um einen >cantus prius factus handelt, macht die Illusion, der formale Prozess von op. 116/5 sei aus der anfänglich exponierten Zelle entwickelt, unmöglich. Wenn schon eine Form von Narrativ unterlegt würde, dann eher das einer Verwandlung. Ein anfänglich präsentierter Zustand stellt sich nach dem Gang durch ein kontrapunktisches Labyrinth als etwas ganz anderes, vorher Verborgenes heraus.

Auch ist hier nicht zu beobachten, dass in der Dramaturgie der Form einem als naturhaft-organisch zu erwartenden motivischen Prozess durch von außen hinzugefügte Symmetrie ein über-natürlicher dialektischer Verlauf eingepflanzt würde, wie auch der 
formal wie harmonisch dissonanteste Moment in den Takten 13-15 weder als Zaudern oder Krise noch als Stau inszeniert wird, sondern einfach seinen Gang nimmt. Vielmehr scheinen von Anfang an geplante und nicht ohne Gewalt vorgenommene symmetrische Maßnahmen die Illusion eines naturhaften Zustandes in den letzten Takten wiederherzustellen.

\section{Synchron und diachron}

Für welches Narrativ man sich bei den drei untersuchten Beispielen auch entscheidet oder ob man überhaupt als Ausgangshypothese einer analytischen Betrachtung narrative Logik unterstellt, hängt von Anlass und Stil der jeweiligen Analyse ab.

Allgemein kann allerdings formuliert werden, dass alle drei Stücke die formalen Bedingungen erfüllen, als Zusammenhang zwischen motivischen Keimzellen und formaler Disposition konzipiert zu sein und auch so hörend nachvollzogen werden zu können, wobei nur Schumanns Choral dies in herkömmlichem Verständnis der organischen Form leisten könnte. Ein Großteil der Zusammenhänge in den anderen Stücken wird analytisch erst dann offenbar, wenn als `Keimzelle nicht nur eine bestimmte rhythmisch-melodische Prägung verstanden wird, die womöglich eröffnend exponiert werden muss, sondern auch die an eine bestimmte Tonhöhenstruktur gebundenen Optionen, die im Verlauf der Form wiedererkennbar entwickelt werden. Da die Ästhetik der ১organic metaphor einem historischen Umfeld entstammt, das kontrapunktische Arbeit grundsätzlich nicht zum Bereich der Erfindung zählte, kommt es bis heute seltener als nötig zu einer Betrachtung im engeren Sinne kontrapunktischer Optionen wie symmetrischer Projektionen und ihrer Interaktion mit Prozessen einer dennoch als organisch verstandenen Form.

Carl Dahlhaus hat in dem sehr kurzen Text zur Mythenanalyse von Claude LéviStrauss $^{51}$ mit den Kernbegriffen synchron und diachron unter anderem auf die Notwendigkeit der Unterscheidung zwischen einer strukturellen Ableitung und einer prozesshaften Struktur hingewiesen, wobei er - wie es scheint - einige missverständliche Konnotationen ${ }^{52}$ einer Theorie der organischen Form sinnvoll relativiert hat. Sogar bei Wagner muss ein zu hörender motivischer Ableitungsprozess durchaus nicht auf eine Substruktur verweisen, die den organischen Zusammenhalt der komponierten Musik wiedergibt. Gerade synchrone Bezüge, die möglicherweise dem analytisch erfassbaren Aufbau einer Komposition und der präzisen Interaktion zwischen motivischem Detail und Großform am nächsten kommen, erklingen häufig nicht als offensichtlicher Prozess. Ebenso wenig muss ein prozessualer Verlauf als Demonstration wachsender Stärke erklingen.

51 Vgl. Dahlhaus 1987b. Zitiert wird vor allem Lévi-Strauss 1976, 30-31.

52 So sicher auch die unaufgearbeiteten Konsequenzen einer zu wenig reflektierten Rezeption Ernst Kurths insofern, als die energetisch durchtränkte Wachstums-, Steigerungs- und Erlebnismetaphorik aus den Inhalten zum Teil gar nicht herauszulösen ist. Vgl. Kurth 1923, 444-571 (= VII. Abschnitt: »Die unendliche Melodie»). 


\section{Literatur}

Adorno, Theodor W. (1970), Ästhetische Theorie (= Gesammelte Schriften 7), hg. von Gretel Adorno und Rolf Tiedemann, Frankfurt a. M.: Suhrkamp.

Bach, Johann Sebastian (1765/69), Johann Sebastian Bachs vierstimmige Choralgesänge, gesammlet von Carl Philipp Emanuel Bach, 2 Teile, Berlin und Leipzig, Reprint Hildesheim u. a.: Olms 1975.

Bolay, Astrid (2013), »Musikalische Analyse im Licht kognitivistischer Forschung. Betrachtungen zu einer Sarabande von Johann Sebastian Bach«, in: Kreativität. Struktur und Emotion, hg. von Andreas Lehmann, Ariane Jeßulat und Christoph Wünsch, Würzburg: Königshausen \& Neumann, 93-100.

Borio, Gianmario (2011), »Organische Form jenseits von Beethoven. Über die Neuorientierung der musikalischen Formenlehre in den 1920er und 1930er Jahren«, in: Ereignis und Exegese. Musikalische Interpretation - Interpretation der Musik. Festschrift für Hermann Danuser zum 65. Geburtstag, hg. von Camilla Bork, Tobias Robert Klein, Burkhard Meischein, Andreas Meyer und Tobias Plebuch, Schliengen: Argus, 149-167.

Bornstein, Andrea (2004), Two-Part Italian Didactic Music. Printed Collections of the Renaissance and Baroque (1521-1744) Bd. 1, Bologna: Ut Orpheus Edizioni.

Burnham, Scott (2002), »Form«, in: Christensen 2002, 880-906.

Caplin, William E. (2002), »Theories of Musical Rhythm in the Eighteenth and Nineteenth Centuries«, in: Christensen 2002, 657-694.

Christensen, Thomas (Hg.) (2002), The Cambridge History of Western Music Theory, Cambridge: Cambridge University Press.

Cone, Edward T. (1974), The Composer's Voice, Ernest Bloch Lectures, Berkeley: University of California Press.

Danuser, Hermann (2014), »Form - Formation - Transformation«, in: ders., Gesammelte Aufsätze und Schriften, hg. von Hans-Joachim Hinrichsen, Christian Schaper und Laure Spaltenstein, Schliengen: Argus, 306-326.

Dahlhaus, Carl (1970), Analyse und Werturteil (= Musikpädagogik. Forschung und Lehre 8), Mainz: Schott.

_ (Hg.) (1974), Das Problem Mendelssohn, Regensburg: Bosse. (1987a), Ludwig van Beethoven und seine Zeit, Laaber: Laaber.

— (1987b), »Musik als strukturelle Analyse des Mythos. Claude Lévi-Strauss und >Der Ring des Nibelungen«", in: Wege des Mythos in der Moderne. Richard Wagner >Der Ring des Nibelungen،. Eine Münchner Ringvorlesung, hg. von Dieter Borchmeyer, München: dtv, 64-74.

(1988), Klassische und romantische Musikästhetik, Laaber: Laaber.

Dahlhaus, Carl / Miller, Norbert (2007), Europäische Romantik in der Musik. Bd. 2: Von E. T. A. Hoffmann zu Richard Wagner 1800-1850 Bd. 2, Stuttgart und Weimar: Metzler. 
Frank, Manfred (1989), „Das Motiv des skalten Herzens in der romantisch-symbolistischen Deutung", in: ders., Kaltes Herz, Unendliche Fahrt, Neue Mythologie, Frankfurt: Suhrkamp, 11-49.

Goethe, Johann Wolfgang (1977), Schriften zur Naturwissenschaft, hg. von Michael Böhler, Stuttgart: Reclam.

_ (1982), Wilhelm Meisters Wanderjahre, hg. von Ehrhard Bahr, Neuausgabe Stuttgart: Reclam.

Hanslick, Eduard (1891), Vom musikalisch Schönen, 8. Auflage, Wiesbaden: Breitkopf \& Härtel.

Hegel, Georg Wilhelm Friedrich (1987), Phänomenologie des Geistes, Neuausgabe mit einem Nachwort von Lorenz Bruno Puntel, Stuttgart: Reclam.

Hübler, Klaus K. (1989), »Die Kunst, ohne Einfälle zu komponieren. Dargestellt an Johannes Brahms' späten ıIntermezzi«", in: Aimez-vous Brahms »the progressive (= MusikKonzepte 65) hg. von Heinz-Klaus Metzger und Rainer Riehn, München: edition text + kritik, 24-40.

Ickstadt, Andreas (2014), »Fortschritt durch Bewahrung. Über die integrierende und dissoziierende Funktion von Imitationstechniken bei Johannes Brahms «, in: Musikalische Logik und musikalischer Zusammenhang, hg. von Patrick Boenke und Birger Petersen, Hildesheim u.a.: Olms, 91-103.

Jelensperger, Daniel (1833), Die Harmonie im Anfange des neunzehnten Jahrhunderts und die Art sie zu erlernen [ $L$ 'harmonie au commencement du dix-neuvième siècle et méthode pour l'etudier, 1830], Leipzig: Breitkopf \& Härtel.

Karl, Gregory / Robinson, Jenefer (1997), „Shostakovitch's Tenth Symphony and the Musical Expression of Cognitively Complex Emotions", in: Music and Meaning, hg. von Jenefer Robinson, Ithaca and London: Cornell University Press, 154-178.

Kirnberger, Johann Philipp (1776/1779), Die Kunst des reinen Satzes in der Musik, 2 Teile, Reprint Hildesheim u. a.: Olms 1968.

Koch, Heinrich Christoph (1793), Versuch einer Anleitung zur Composition, 3. Teil, Leipzig: Böhme.

Komar, Arthur (1971), Dichterliebe. An Authoritative Score, New York: Norton \& Company.

Krummacher, Friedhelm (1980), »Die Synthesis des Disparaten - Zu Beethovens späten Quartetten und ihrer frühen Rezeption«, AfMw XXXVII, 99-134.

Kühn, Clemens (1993), Analyse lernen (= Bärenreiter Studienbücher Musik 4), Kassel u. a.: Bärenreiter.

Kurth, Ernst (1923), Romantische Harmonik und ihre Krise in Wagners »Tristan«, Reprint Hildesheim u. a.: Olms 1998.

Lakoff, George / Johnson, Mark (1997), Leben in Metaphern. Konstruktion und Gebrauch von Sprachbildern, Heidelberg: Carl-Auer-Systeme.

Lange, Thomas (2010), Das bildnerische Denken Philipp Otto Runges, Berlin, München: Deutscher Kunstverlag. 
Lévi-Strauss, Claude (1976), Mythologica I. Das Rohe und das Gekochte, Frankfurt a. M.: Suhrkamp.

Lobe, Johann Christian (1858), Lehrbuch der musikalischen Komposition, zweite verbesserte Auflage, Bd. 1, Leipzig: Breitkopf \& Härtel.

Lorenz, Alfred (1924), Das Geheimnis der Form bei Richard Wagner. Bd. 1: Der musikalische Aufbau des Bühnenfestspiels Der Ring des Nibelungen, Reprint Tutzing: Schneider 1966.

Ludwig, Ralf (1997), Hegel für Anfänger. Phänomenologie des Geistes, München: dtv.

Marx, Adolf Bernhard (1825), Rez. „Sonate für das Pianoforte, über die Figur [...] komponiert von Ludwig Berger von Berlin [...],«, Berliner allgemeine musikalische Zeitung 2, 399-400.

Poos, Heinrich (1995), Johann Sebastian Bach. Der Choralsatz als musikalisches Kunstwerk (= Musik-Konzepte 87), hg. von Heinz-Kaus Metzger und Rainer Riehn, München: edition text + kritik.

Ratz, Erwin (1973), Einführung in die musikalische Formenlehre, dritte, erweiterte und neugestaltete Aufl., Wien: Universal Edition.

Reti, Rudolph (1961), The Thematic Process in Music, London: Faber \& Faber.

Rohringer, Stefan (2014), »Metapher und musikalische Analyse», in: Musikalische Analyse. Begriffe, Geschichten, Methoden (= Grundlagen der Musik 8), hg. von Felix Diergarten, Laaber: Laaber, 161-184.

Schönberg, Arnold (1979), Die Grundlagen der musikalischen Komposition, ins Deutsche übertragen von Rudolf Kolisch, hg. von Rudolf Stephan, Wien: Universal Edition.

_ (1992), »Brahms, der Fortschrittliche«, in: ders., Stil und Gedanke (= Gesammelte Schriften 1), hg. von Ivan Vojtech, Frankfurt a. M.: Fischer, 54-104.

Schumann, Robert (1982), Schriften über Musik und Musiker, hg. von Josef Häusler, Stuttgart: Reclam.

Sulzer, Johann Georg (1771), Allgemeine Theorie der Schönen Künste, Bd. 1, Leipzig: Weidemanns Erben und Reich.

Thorau, Christian (2012), Vom Klang zur Metapher. Perspektiven der musikalischen Analyse (= Studien und Materialien zur Musikwissenschaft 71), Hildesheim u. a.: Olms.

Weber, Gottfried (1817), Versuch einer geordneten Theorie der Tonkunst zum Selbstunterricht mit Anmerkungen für Gelehrtere, Bd. 1, Mainz: Schott. 